\title{
Fingerprint Matching using A Hybrid Shape and Orientation Descriptor
}

\author{
Joshua Abraham ${ }^{1}$, Paul Kwan ${ }^{2}$ and Junbin $\mathrm{Gao}^{3}$ \\ ${ }^{1}$ School of Chemistry and Forensic Science, University of Technology Sydney \\ ${ }^{2}$ School of Science and Technology, University of New England \\ ${ }^{3}$ School of Computing and Mathematics, Charles Sturt University \\ Australia
}

\section{Introduction}

Minutiae-based methods have been used in many commercial fingerprint matching systems. Based primarily on a point pattern matching model, these methods rely heavily on the accuracy of minutiae extraction and the detection of landmarks like core and delta for pre-alignment. Spurious and missing minutiae can both introduce errors in minutiae correspondence. Equally problematic is the inability to detect landmarks to guide pre-alignment. Taken together, these problems lead to sub-optimal matching accuracy.

Fortunately, the contextual information provided by ridge flow and orientation in the neighborhood of detected minutiae can help eliminate spurious minutiae while compensating for the absence of genuinely missing minutiae both before and during matching. In addition, coupled with a core detection algorithm that can robustly handle missing or partially available landmarks for pre-alignment, significant improvement in matching accuracy can be expected. In this chapter, we will firstly review fingerprint feature extraction, minutiae representation, and registration, which are important components of fingerprint matching algorithms. Following this, we will detail a relevant fingerprint matching algorithm based on the Shape Context descriptor found in Kwan et al. (2006). Next, we will introduce a novel hybrid shape and orientation descriptor that is designed to address the above problems. The hybrid descriptor can effectively filter out spurious or unnatural minutiae pairings while simultaneously using the additional ridge orientation cues in improving match score calculation. In addition, the proposed method can handle situations where either the cores are not well defined for detection or the fingerprints have only partial overlapping. Lastly, experiments conducted on two publicly available fingerprint databases confirm that the proposed hybrid method outperforms other methods included in our performance comparison.

\subsection{Fingerprint recognition}

An essential component of Automated Fingerprint Recognition Systems (AFRS) is the matcher module which makes use of fingerprint matching algorithms in order to match a test fingerprint against template fingerprint(s) for identification/verification (see Figure 1). Currently, reliable fingerprint matching is a non-trivial problem due to environmental noise and uniqueness of each impression. The accuracy of fingerprint matching algorithms depends 
on the image quality, image enhancement methods, feature set extraction algorithms, and feature set pre-processing/post-processing algorithms.

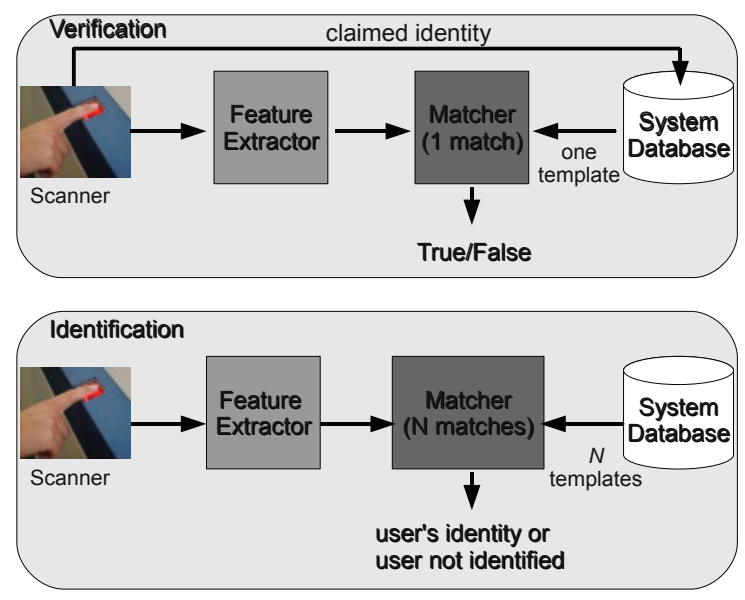

Fig. 1. Basic models for fingerprint verification and identification processes.

Noisy features introduced from environmental factors such as dust, scars, skin dryness, and scaring, are strongly desired to be removed or kept to a minimal level. Even highly robust matching algorithms will suffer from poor matching performance when inaccurate feature extraction and filtering, high noise, poor image quality, or undesirable effects from image enhancement occur.

Even without the advent of environmental noise, applied impressions of the same fingerprint are not guaranteed to be identical due to variability in displacement, rotation, scanned regions, and non-linear distortion or 'warping'. Displacement, rotation, and disjoint detected regions are obviously due to the differences in the physical placement of a finger on a scanner. Figure 2 shows different impressions of the same finger and the noticeable variability in the mentioned areas. One aspect that may be harder to see with the naked eye is non-linear distortion, which is due to both skin elasticity and angular and force variability in applied pressure.

Fingerprint matching algorithm largely follow 3 different classes: correlation-based, minutiae-based, and non-minutiae feature based matching. Correlation-based matching (such as Hatano et al. (2002) and Lindoso et al. (2007)) involves superimposing 2 fingerprint images together and calculating pixel-wise correlation for different displacement and rotations. Minutia-based matching uses extracted minutiae from both fingerprints in order to help perform alignment and retrieve minutiae pairings between both fingerprint minutiae sets. Minutiae-based matching can be viewed as a point-pattern matching problem with theoretical roots in pattern recognition and computer vision. Non-minutiae feature based matching (for example Yang \& Park (2008) and Nanni \& Lumini (2009)) use non-minutiae features, such as ridge shape, orientation and frequency images in order to perform alignment and matching. Amongst all algorithm classes, minutiae-based methods are the most common due to their strict analogy with the way forensic experts compare fingerprints and legal acceptance as a proof of identity in many countries (Ratha \& Bolle, 2003). Minutiae points are also known to be extremely unique from finger to finger in terms of spatial distribution, proving to be ideal features for fingerprint matching. Additionally, minutiae point sets obtain a higher level of 

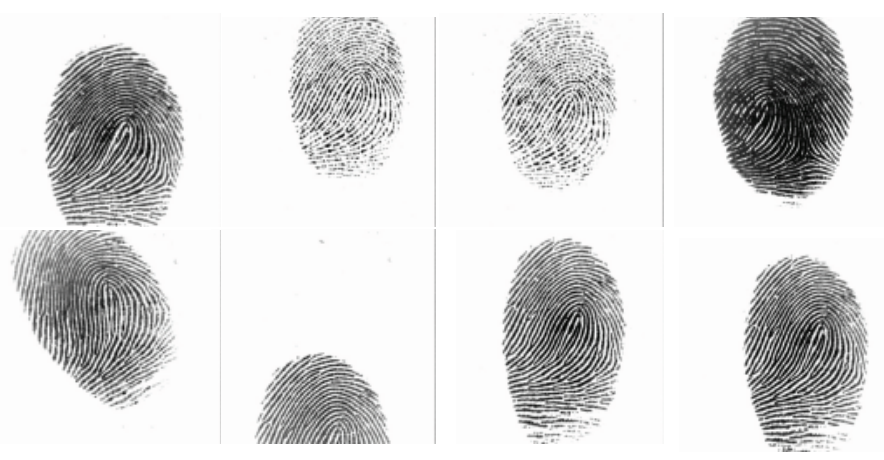

Fig. 2. Eight impressions of the same fingerprint from the FVC2002 database (Maio et al., 2002) with noticeable differences in region overlap, offset, orientation, and image quality.)

uniqueness versus practicality in comparison to other level types of fingerprint features, such as ridge orientation/frequency images and skin pores.

\section{Base theory}

\subsection{Minutiae extraction}

Since the vast majority of fingerprint matching algorithms rely on minutiae matching, minutiae information are regarded as highly significant features for AFRS. The two main methods of minutiae feature extraction either require the gray-scale image to be converted to a binary image, or work directly on a raw or enhanced gray-scale image.

In the binary image based method, the binarization of the gray-scale image is the initial step. This requires each gray-scale pixel intensity value to be transformed to a binary intensity of black (0) or white (1). The simplest approach is to apply a global threshold where each pixel is mapped according to

$$
I(x, y)= \begin{cases}1 & \text { if } I(x, y) \geq t \\ 0 & \text { otherwise }\end{cases}
$$

Although novel, this method is usually not adequate since fingerprint images may have differing levels of contrast throughout the image. However, the same method can be applied with locally adaptive thresholds. Other more advanced approaches include ridge/valley edge detection techniques using Laplacian operators as in Xiao \& Raafat (1991), and mathematical morphology in Gonzalez \& Woods (2007).

Once produced, the binary image usually undergoes a morphological thinning operation, where ridge structures are reduced to 1-pixel thickness, referred to as the skeleton, in order to aid minutiae detection. The resulting thinned binary image then has each pixel, $\mathbf{p}$, analysed in order to find minutiae location. This is achieved by having the 8-neighbourhood (pixels within $3 \times 3$ window centred at $\mathbf{p}$ ) circularly traversed in an anti-clockwise manner in order to produce the Rutovitz crossing number introduced in Rutovitz (1966) 


$$
c n(\mathbf{p})=\frac{1}{2} \sum_{i=1 \ldots 8}\left|\operatorname{val}\left(\mathbf{p}_{(i \bmod 8)}\right)-\operatorname{val}\left(\mathbf{p}_{i-1}\right)\right|
$$

where val $\in\{0,1\}$ (i.e. binary image pixel intensity value). Minutiae pixel locations can now be identified, as ridge endings will have $c n=1$ and ridge bifurcations will have $c n=3$.
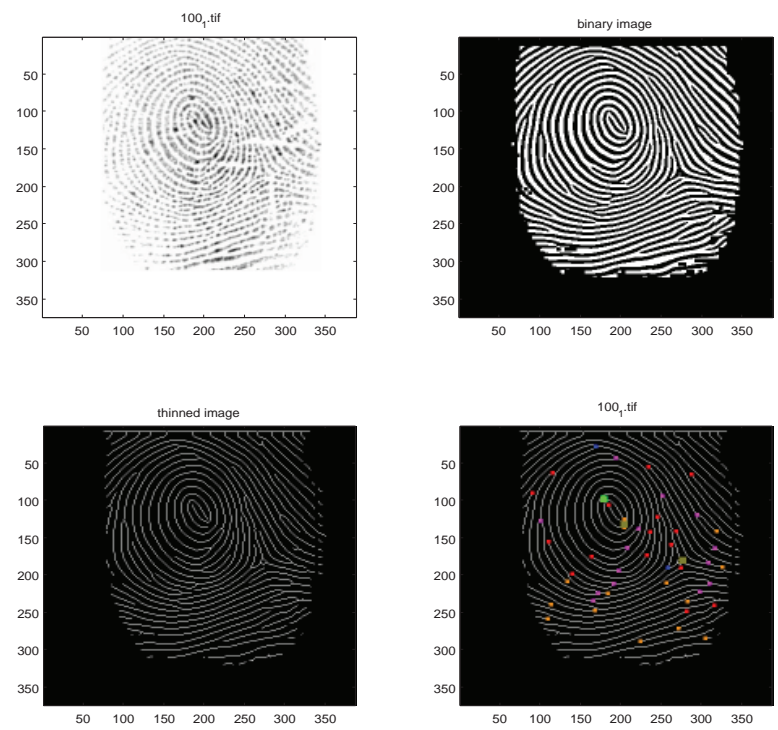

Fig. 3. top left: Original gray-scale image. top right: binary image bottom left: inverted skeleton (thinned) image bottom right: inverted skeleton image with core point (green), delta/lower core points (gold), bifurcations (blue for $\theta \in\left[0^{\circ}-180^{\circ}\right.$ ) and purple for $\theta \in\left[180^{\circ}-360^{\circ}\right)$, and ridge endings (orange for $\theta \in\left[0^{\circ}-180^{\circ}\right.$ ) and red for $\theta \in\left[180^{\circ}-360^{\circ}\right)$.

Although binarization in conjunction with morphological thinning provides a simple framework for minutiae extraction, there are a couple of problematic characteristics. Spurious minutiae (false minutiae) due to thinning algorithms (such as spurs) or irregular ridge endings. Additionally, performance is also an issue since binarization and specifically morphological thinning algorithms are known to be computationally expensive (see Figure 3).

Direct gray-scale minutiae extraction attempts to overcome the problems introduced by image binarization and thinning. One key gray-scale based method that the algorithm in Maio \& Maltoni (1997) employs is ridge path following, where an initial point $\left(x_{1}, y_{1}\right)$ has a $k$ pixel length path projected toward an initial direction, $\theta_{1}$, and likewise, subsequent iterations have the base point $\left(x_{t_{n}}, y_{t_{n}}\right)$ project the next ridge sample point $\left(x_{n+1}, y_{n+1}\right)$ in the direction $\theta_{n}$. Analysis of the section set $S_{n}$, being a 1 dimensional cross section slice centred about $\left(x_{t_{n}}, y_{t_{n}}\right)$ and orthogonal to $\theta_{n}$ with length $2 \sigma+1$ where $\sigma$ is the average thickness of a ridge, is used to retrieve $\theta_{n}$, and ultimately, $\left(x_{n+1}, y_{n+1}\right)$. The path following algorithm terminates when a local maxima cannot be found at the current point's section set, giving clear indication that a ridge ending or bifurcation is reached. 
In Farina et al. (1999), these structures and others were removed from the skeleton image. Minutiae were also categorised or ranked according to the degree of their meeting defined topological rules. A similar approach was used in Zhao \& Tang (2007), where dot (isolated pixel) filtering, small holes filling (i.e. possibly from dominant pores) were used, in combination with other heuristics. The accuracy of a fingerprint matching algorithm was reported to be decreased by approximately $13.5 \%$ when minutiae filtering heuristics were used in comparison to no filtering.

\subsection{Minutiae representation}

Minutiae-based matching algorithms are largely dependent on extracted minutiae information. Robust minutiae-based matching algorithms have to deal with occurrences of missing and spurious minutiae, where missing minutiae can occur as a result of inaccurate feature detection, feature post-processing, or image noise obscuring minutiae detail, and spurious minutiae can be introduced by dry skin, creases, feature detection algorithms, and other potential noise causing agents. The general processes of a fingerprint matching algorithm is presented in Figure 4.

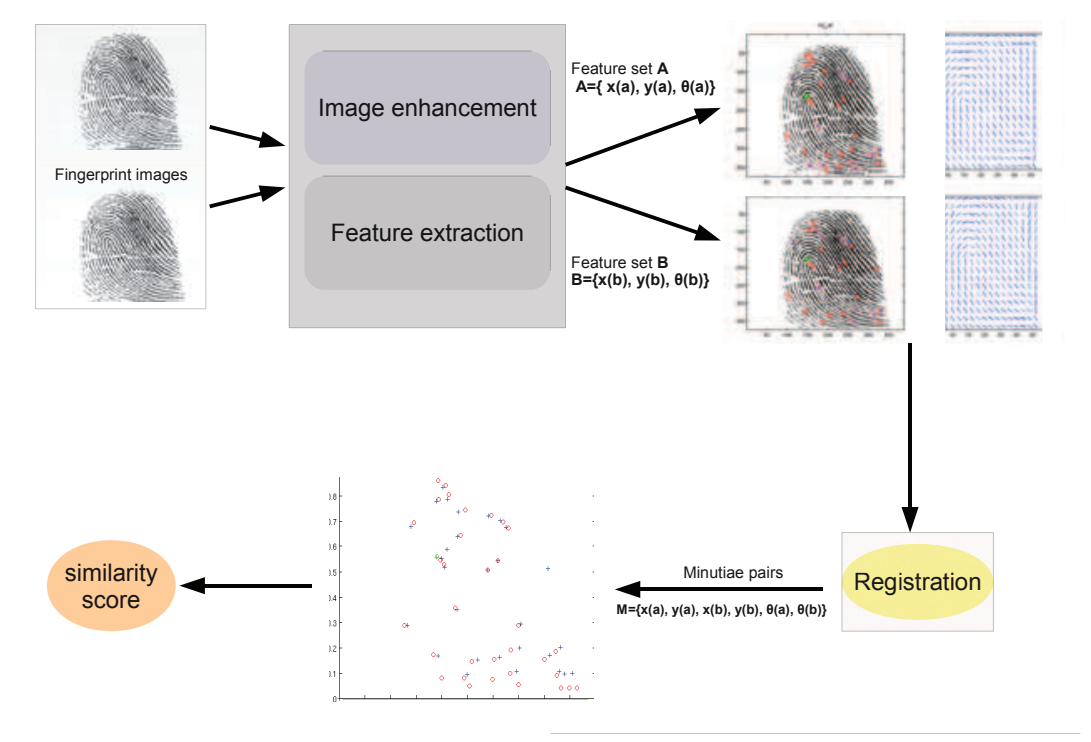

Fig. 4. General processes for minutiae-based fingerprint matching.

In minutiae-based matching, minutiae are commonly represented as minutiae structures called minutia triplets, where a minutia, $m_{i}$, is described as $m_{i}=\{x, y, \theta\}$ with $x, y$ representing the $\mathrm{x}-\mathrm{y}$ coordinate of the minutia and $\theta$ the angular direction of the main ridge (see Figure 5 left).

The main focus of minutiae-based matching is to perform a one-to-one mapping or pairing of minutiae points from a test image minutiae set

$$
A=\left\{m_{A_{1}}, m_{A_{2}}, \ldots, m_{A_{p}}\right\}, \text { where } m_{A_{i}}=\left\{x_{A_{i}}, y_{A_{i}}, \theta_{A_{i}}\right\} \text { and } 1 \leq i \leq p
$$


to a template image minutiae set

$$
B=\left\{m_{B_{1}}, m_{B_{2}}, \ldots, m_{B_{q}}\right\} \text {, where } m_{B_{j}}=\left\{x_{B_{j}}, y_{B_{j}}, \theta_{B_{j}}\right\} \text { and } 1 \leq j \leq q,
$$

forming the minutiae pairs $\left(m_{A_{k}}, m_{B_{\pi(k)}}\right)$ with $\pi(k)$ as the mapping permutation of pairs from set $A$ to $B$.

Unfortunately, we cannot proceed to find minutiae pairs from triplets without some pre-processing for the following critical reasons:

- fingerprint impressions can differ in orientation, deeming the direction field in the triplet useless,

- fingerprint impressions can differ in offset, deeming the $x-y$ fields in the triplet useless, and

- skin elasticity creates non-linear distortion or 'warping' to occur when different directional pressure is applied causing triplet $x-y$ variations to occur.

In general, the lack of invariant characteristics of the triplet structure prohibits it to aid the process of finding minutiae pairs.

\subsection{Registration}

In order to address the issues concerning the lack of invariance of the triplet structure, global registration is required. Global registration concerns the alignment and overlay of the template and test fingerprints so that corresponding regions of the fingerprints have minimal geometric distance to each other. Registration can be achieved geometrically by applying (to either the test or template fingerprint minutiae set) a heuristically guided affine transform, where minutiae triplet field values are updated with

$$
\left[\begin{array}{l}
x_{\text {new }} \\
y_{\text {new }}
\end{array}\right]=\left[\begin{array}{cc}
\cos \left(\theta_{\Delta}\right) & -\sin \left(\theta_{\Delta}\right) \\
\sin \left(\theta_{\Delta}\right) & \cos \left(\theta_{\Delta}\right)
\end{array}\right]\left[\begin{array}{l}
x \\
y
\end{array}\right]+\left[\begin{array}{l}
x_{\Delta} \\
y_{\Delta}
\end{array}\right]
$$

and

$$
\theta_{\text {new }}=\theta-\theta_{\Delta} \text {, }
$$

where $\theta_{\Delta}$ is the orientation difference and $\left(x_{\Delta}, y_{\Delta}\right)$ is the displacement difference in order to super-impose one fingerprint impression on top of the other with accurate overlap and uniform direction.

Even with the advent of high distortion, minutiae points within a fingerprint image are still expected to keep their general global location in relation to the majority of other minutiae points and other key landmarks (such as cores and deltas) when alignment is achieved. Specifically speaking, the spatial distribution or geometric properties of neighbouring minutiae should have minimal difference even in distorted images. If we consider that there are clear limitations in terms of minutiae landmark relative to positioning variability (even with high distortion), while recognising that different fingerprint impressions have orientation and displacement differences, then the global registration process notably reduces the search space. This reduces algorithm complexity for finding minutiae pairs, since matching pairs are formed in smaller local neighbourhoods (i.e. constraints added for minutiae mappings) once aligned. This allows a naive brute force minutiae pairing process to be avoided. 
Following the registration process, we can now produce geometric constraints for the discovery of minutiae matching pairs, including geometric distance:

$$
\operatorname{dist}_{r}\left(m_{A_{i}}, m_{B_{j}}\right)=\sqrt{\left(x_{A_{i}}-x_{B_{j}}\right)^{2}+\left(y_{A_{i}}-y_{B_{j}}\right)^{2}}<r_{\delta},
$$

or to account for scale difference (i.e. if we are comparing images collected from different resolution scanners)

$$
\operatorname{dist}_{r}\left(m_{A_{i}}, m_{B_{j}}\right)=\sqrt{\left(x_{A_{i}}-k_{x} \cdot x_{B_{j}}\right)^{2}+\left(y_{A_{i}}-k_{y} \cdot y_{B_{j}}\right)^{2}}<r_{\delta},
$$

and minutiae angle difference,

$$
\operatorname{dist}_{\theta}\left(m_{A_{i}}, m_{B_{j}}\right)=\min \left(\left|\theta_{A_{i}}-\theta_{B_{j}}\right|, 360^{\circ}-\left|\theta_{A_{i}}-\theta_{B_{j}}\right|\right)<r_{\theta} .
$$

The geometric tolerance $r_{\delta}$ is in place to account for distortion that may occur, whereas $r_{\theta}$ is the tolerance for angular differences that may arise due to orientation estimations from the ridge orientation images. Following global registration, a local search can now be performed, in order to match minutiae in the $\delta$-neighbourhood that meet the constraints in equations 7-9 (see Figure 5 right).

Once genuine minutiae pairs are produced, a metric of similarity, usually called the similarity score, can then be calculated. The similarity score must accurately describe how similar two fingerprints are, taking into account all of the relevant information obtained from earlier stages, such as number of genuine minutiae pairs and how similar each pair is. One similarity score given in Liang \& Asano (2006) is defined as

$$
\operatorname{sim}(A, B)=\frac{n_{\text {match }}^{2}}{n_{A} n_{B}}
$$

where $n_{\text {match }}$ is the number of matching minutiae pairs, and $n_{A}, n_{B}$ are the number of minutiae in the overlapped regions of the template and test fingerprints following registration.

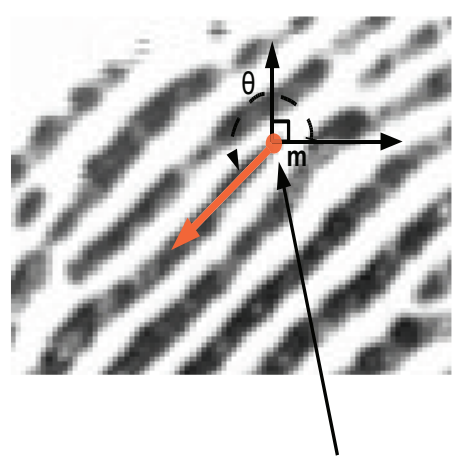

$[x, y]$

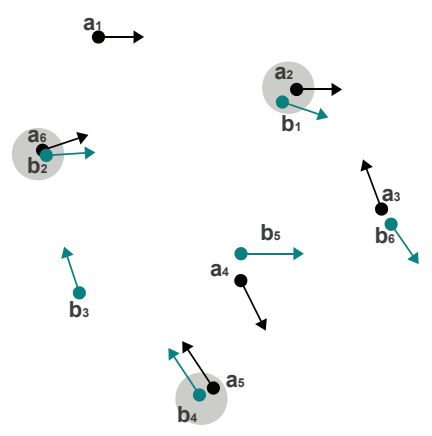

Fig. 5. left: minutia triplet structure representation. right: Minutiae points from 2 different fingerprints being mapped after registration, with gray circles representing pairs with constraints upheld (equations 7-9). 
In order to effectively match fingerprints, we require that the registration used not only be computationally sound, but also perform accurate alignment. In order to achieve this, some methods use additional features (sometimes in combination with minutiae detail) for global alignment, such as cores and deltas, local or global orientation field / texture analysis, and ridge feature analysis.

Using core points for registration is known to dramatically improve the performance of a matching algorithm. In Chikkerur \& Ratha (2005), a graph theory based minutiae matching algorithm reported a $43 \%$ improvement in efficiency when including the core point for registration, without adverse effect toward matching accuracy. In Zhang \& Wang (2002) core points were used as key landmarks for registration. This method proved to be extremely efficient in comparison to other key registration methods. Structural features of minutiae close to the core are used to calculate the rotation needed. The core point was also used in Tian et al. (2007) for registration with the orientation that produced the minutiae pair with the minimum hilbert scanning distance. These and similar methods heavily rely on the core point for alignment. Such a dependence is not strictly robust, since not all fingerprint impressions contain core points and the inclusion of noises may effect the accuracy of core detection algorithms, possibly resulting in incorrect alignments.

In Yager \& Amin (2005), the global orientation image with points divided into hexadecimal cells (see Figure 6 right) is used for registration. The steepest descent algorithm was used in order to find the affine transform $\left(x_{\Delta}, y_{\Delta}, \theta_{\Delta}\right)$ that minimise the cost function

$$
C\left(P, Q^{\prime}\right)=\frac{1}{N} \sum_{p \in P, q^{\prime} \in Q^{\prime}} \min \left[\left(p-q^{\prime}\right),\left(q^{\prime}-p+\pi\right)\right],
$$

where $\mathrm{P}$ is orientation image of one fingerprint and $\mathrm{Q}^{\prime}$ is the orientation image of the second fingerprint following an affine transformation.
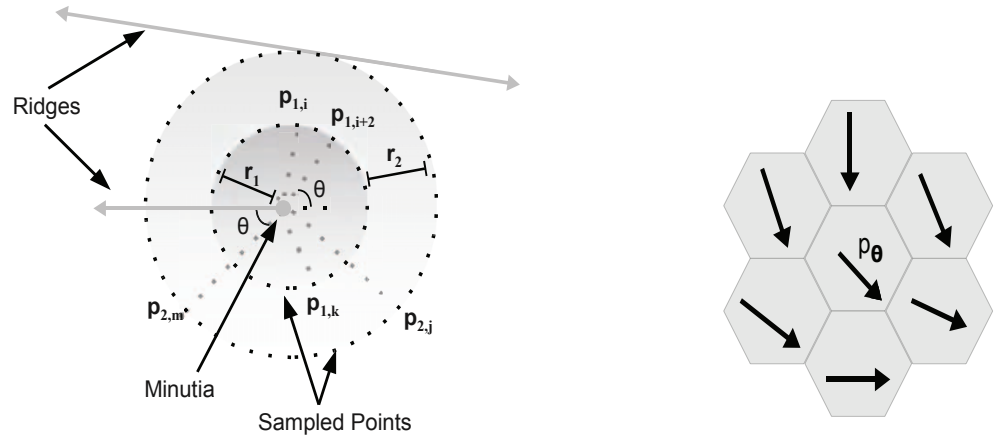

Fig. 6. left: The local orientation descriptor used in Tico \& Kuosmanen (2003). right: Hexagonal orientation cells within the orientation image in Yager \& Amin (2005) .

Another example which uses the global orientation image for registration can be found in Liu et al. (2006). For all possible transforms of the test fingerprint onto the template fingerprint 
which has significant region overlap, the normalised mutual information (NMI) defined as

$$
\operatorname{NMI}(X, Y)=\frac{H(X)+H(Y)}{H(X, Y)}
$$

is calculated, where

$$
\begin{aligned}
H(X) & =-E_{X}[\log P(X)], \\
H(Y) & =-E_{Y}[\log P(Y)],
\end{aligned}
$$

and

$$
H(X, Y)=-E_{X}\left[E_{Y}[\log P(X, Y)]\right],
$$

where $X$ and $Y$ are discrete random variables representing the orientation fields, $O_{x}$ and $O_{y}$, of the template and test fingerprints, respectively, which are divided into $b$ blocks. The probabilities can be calculated as

$$
\begin{gathered}
P_{X Y}(x, y)=\frac{n(x, y)}{\sum_{i=0}^{n-1} \sum_{j=0}^{n-1} n(i, j)}, \\
P_{X}(x)=\sum_{j=0}^{b-1} P(x, j), \\
P_{Y}(y)=\sum_{i=0}^{b-1} P(i, y),
\end{gathered}
$$

and

$$
n(x, y)= \begin{cases}1 & \text { if }\left|O_{x}(x)-O_{x}(y)\right| \leq \lambda \\ 0 & \text { otherwise }\end{cases}
$$

with $\lambda$ as a small threshold, indicating that orientation corresponding image blocks have very similar orientations. We can now find the transform which produces the maximum NMI as the global registration.

Global landmarks and features are not only used for aiding registration. Local structure sets or descriptors can also be used for registration. For instance, in Tico \& Kuosmanen (2003), the rotation and translation invariant minutia orientation descriptor (see Figure 6 Left) is used to find minutiae pair with the maximum probabilistic value

$$
[r, s]=\arg \max _{i, j} P\left(m_{A_{i}}, m_{B_{j}}\right)
$$

with

$$
P\left(m_{A_{i}}, m_{B_{j}}\right)=\frac{S\left(m_{A_{i}}, m_{B_{j}}\right)^{2}}{\left(\sum_{k=1}^{p} S\left(m_{A_{k}}, m_{B_{j}}\right)+\sum_{l=1}^{q} S\left(m_{A_{i}}, m_{B_{l}}\right)\right)}
$$

and $S\left(m_{A_{i}}, m_{B_{j}}\right)$ is the similarity function defined as

$$
S\left(m_{A_{i}}, m_{B_{j}}\right)=(1 / K) \sum_{c}^{L} \sum_{d}^{K_{c}} \exp \left(-\frac{2\left(\min \left(\left|\theta_{c, d}^{A_{i}}-\theta_{c, d}^{B_{j}}\right|, \pi-\left|\theta_{c, d}^{A_{i}}-\theta_{c, d}^{B_{j}}\right|\right)\right)}{\pi \mu}\right)
$$

where the orientation descriptor has a total of $K$ sample points distributed as $L$ concentric circles having $K_{c}$ points (i.e. possibly differing number per circle) with equidistant angular 
distribution (i.e. $\frac{2 \pi}{K_{c}}$ step size), $\theta_{c, d}^{A_{i}}$ is minimum angle required to rotate the $d^{\text {th }}$ sample orientation on $c^{\text {th }}$ circle to the orientation of minutia $m_{A_{i}}$ (likewise for $\theta_{c, d}^{B_{j}}$ ), and $\mu$ is an empirically chosen parameter. After finding the maximum pair index, $[r, s]$, the affine transform is performed on the set $B=\left\{m_{B_{1}}, m_{B_{2}}, \ldots, m_{B_{q}}\right\}$, with $\theta_{\Delta}=\theta_{A_{r}}-\theta_{B_{s}}$ and $\left[\begin{array}{ll}x_{\Delta} & y_{\Delta}\end{array}\right]^{T}=\left[\begin{array}{ll}x_{A_{r}}-x_{B_{s}} & y_{A_{r}}-y_{B_{s}}\end{array}\right]^{T}$ as the transformation parameters. The additional local texture information contained in the orientation-based descriptor is then used in the similarity score to give

$$
\operatorname{sim}(A, B)=\frac{\left(\sum_{(i, j) \in C} S\left(m_{A_{i}}, m_{B_{j}}\right)\right)^{2}}{n_{A} n_{B}}
$$

where $S\left(m_{A_{i}}, m_{B_{i}}\right)$ is the function defined in equation $22, C$ is the set of minutiae pairs, and $A_{i}, B_{j}$ are the template/test minutiae list indexes, respectively.

Unlike most algorithms that have global registration preceding local registration or minutiae pairing, the proposed method in Bazen \& Gerez (2003) finds a list of minutiae pairs prior to performing global registration. Each minutia in the template and test fingerprints have an extended triplet structure defined as a 2-neighbourhood structure in the form of $\left\{x, y, \theta, r_{1}, \theta_{1}, r_{2}, \theta_{2}\right\}$, where $r_{1}$ and $\theta_{1}$ are the polar co-ordinates of the closest minutia, and likewise for the second closest minutia, $r_{2}$ and $\theta_{2}$. The list is then built by finding pairs from after aligning each minutiae structure and then comparing the similarity. This initial minutiae list may contain false pairs. Using the largest group of pairs that use approximately the same transform parameters for alignment, a least squares approach is then used to find the optimal registration. To aid highly distorted fingerprints the non-affine transformation model based on the Thin Plate Spline (T.P.S) (defined in section 3.1.1) is applied to model distortion, with minutiae pair correspondences as anchor points. Such a model allows the minutiae pair restrictions of equations 7-9 to be more rigorously set, helping reduce an algorithms FAR (False Accentance Rate).

There exist algorithms that bypass global registration all together. In Chikkerur \& Govindaraju (2006), a proposed local neighbourhood minutia structure called K-plet uses a graph theory based consolidation process in combination with dynamic programming for local matching (i.e. minutiae pairing). Another example of a matching algorithm that does not require registration can be found in Kisel et al. (2008), which opts to use translation invariant minutia structures with neighbourhood information for finding genuine minutiae correspondences.

For the majority of algorithms that use global registration, local minutiae matching is then performed. In order to aid local matching, structures based on triplets and other shape descriptors, which are shape descriptive data sets employed for the geometric analysis of shapes (that may have been previously utilised in the registration process), can be used to measure minutiae similarity. For instance, a greedy algorithm is used in Tico \& Kuosmanen (2003), where subsequent pairs are selected in order of descending probability values (i.e. equation 21) in conjunction with equations 7-9. A similar methodology can be found in Qi et al. (2004), where a greedy algorithm and textural minutia-based descriptor is similarly used.

\section{Hybrid shape and orientation descriptor}

In this section, a brief theoretical foundation concerning the Thin Plate Spline (T.P.S) and shape context will initially be established. Following this, a fingerprint matching algorithm using 
the enhanced shape context descriptor introduced in Kwan et al. (2006) is reviewed. Next, the proposed hybrid descriptor method which uses the enhanced shape context descriptor in conjunction with the orientation-based descriptor described in Tico \& Kuosmanen (2003) will be introduced. Experimental results will be reported in Section 3.2.3.

\subsection{Enhanced shape context}

Shape matching algorithms that use contour based descriptors based on point samples (or point pattern matching algorithms) are analogous to minutiae-based fingerprint matching algorithms, as they usually combine the use of descriptors with dynamic programming, greedy, simulated annealing, and energy minimization based algorithms, in order to register shapes and compute a similarity measure. Hence, like fingerprint matching algorithms, desirable characteristics of shape matching methods are invariance to rotation and scale, while achieving robustness toward small amounts of distortion and outlier point samples.

The Shape Context descriptor in Belongie et al. (2000) and Belongie et al. (2002) is a robust contour based shape descriptor used for calculating shape similarity and the recovering of point correspondences. Recently, Kwan et al. (2006) proposed a fingerprint matching based variant of the shape context, the Enhanced Shape Context, utilising additional contextual information from minutiae sets.

Initially, we are given $n$ and $m$ minutiae from test and template fingerprints, $P$ and $Q$, respectively. For each minutia, $p_{i} \in P$, we are to find the best matching minutia, $q_{j} \in Q$. When the shape context descriptor is constructed for a particular minutia, a coarse histogram

$$
h_{p_{i}}(k)=\#\left\{p_{j} \neq p_{i}:\left(p_{j}-p_{i}\right) \in \operatorname{bin}(k)\right\} .
$$

involving the remaining $n-1$ minutiae of $P$ is built as the shape context of minutia $p_{i}$. Each bin corresponds to the tally of minutiae in a particular spatial region with distance $r_{l} \leq d \leq r_{h}$ and direction $\theta$.

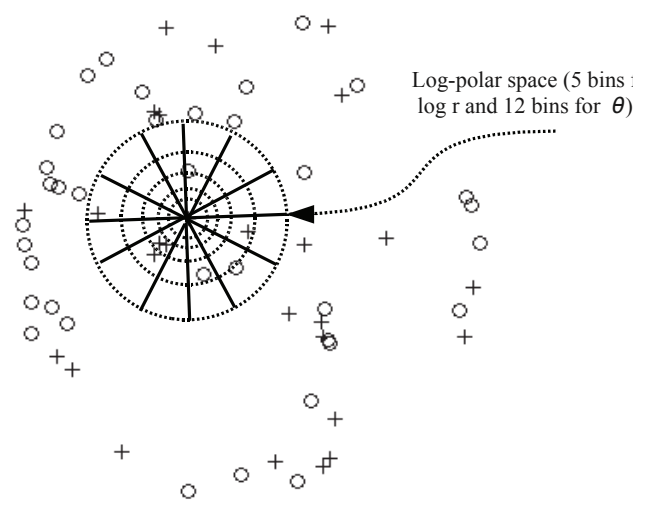

Fig. 7. Log-polar histogram bins used to create shape context histogram for a minutia point. Bifurcations and ridge endings are denoted $\mathrm{by}^{\prime}+$ ' and ' $\mathrm{o}$ ', respectively.

The spatial geometric regions are divided to be uniform in log-polar space, where the log-polar transformation is defined as the mapping from the Cartesian plane $(x, y)$ to the log-polar plane $(\xi, \eta)$ with 


$$
\left[\begin{array}{l}
\xi \\
\eta
\end{array}\right]=\left[\begin{array}{c}
\log r \\
\theta
\end{array}\right]=\left[\begin{array}{c}
\log \sqrt{x^{2}+y^{2}} \\
\arctan \frac{y}{x}
\end{array}\right] .
$$

The shape context descriptor is then constructed for each minutiae $p_{i} \in P$, and likewise, each $q_{j} \in Q$, providing a localised spatial survey of the minutiae distributions for each fingerprint. We can now consider the cost of matching two minutiae, which we can later use to find the optimal mapping of minutiae. Let $C_{i j}=C\left(p_{i}, q_{j}\right)$ denote the cost of matching minutia $p_{i} \in P$ with $q_{j} \in Q$.

Since the shape context are distributed as histograms, we can use a modification of the $\chi^{2}$ statistic:

$$
C_{i j} \equiv C\left(p_{i}, q_{j}\right)=\frac{1}{2} \sum_{k=1}^{K} \frac{\left[h_{p_{i}}(k)-h_{q_{j}}(k)\right]^{2}}{h_{p_{i}}(k)+h_{q_{j}}(k)}
$$

where $h_{p_{i}}$ and $h_{q_{j}}$ denote the $K$-bin histograms of points $p_{i}$ and $q_{j}$, respectively. This cost can be modified to include application specific weighting and additional costs, in order to add extra relevant information, and hence, improve accuracy.

In order to improve overall accuracy, the enhanced shape context cost value was modified to include contextual information, such as minutia type (i.e., bifurcation and ridge endings, as shown in Figure 7) and minutia angle. This produced the modified log-polar histogram cost as

$$
C_{i j}^{*} \equiv C^{*}\left(p_{i}, q_{j}\right)=\left(1-\gamma C_{i j}^{\text {type }} C_{i j}^{\text {angle }}\right) \cdot\left(\frac{1}{2} \sum_{k=1}^{K} \frac{\left[h_{p_{i}}(k)-h_{q_{j}}(k)\right]^{2}}{h_{p_{i}}(k)+h_{q_{j}}(k)}\right)
$$

with $0 \leq \gamma \leq 1$,

$$
C_{i j}^{\text {type }}= \begin{cases}-1 & \text { if type }\left(p_{i}\right)=\operatorname{type}\left(q_{j}\right) \\ 0 & \text { if } \operatorname{type}\left(p_{i}\right) \neq \operatorname{type}\left(q_{j}\right)\end{cases}
$$

and

$$
C_{i j}^{\text {angle }}=-\frac{1}{2}\left(1+\cos \left(\left(\angle_{\text {initial-warped }}\right)\right)\right)
$$

where $\angle_{\text {initial-warped }}$ is the absolute value of the angle difference in the ridge orientation tangent at $p_{i}$ and $q_{j}$ in the beginning and after each iterative warping (see section 3.1.1). If $\angle_{\text {initial-warped }}$ is greater than $\pi$, it is adjusted as $2 \pi-\angle_{\text {initial-warped }}$ so it will less than or equal to $\pi$.

After computing the cost $C_{i j}^{*}$ for all possible $n \times m$ pairs, the mapping permutation (one-to-one), $\pi$, that minimises the total matching cost

$$
H(\pi)=\sum_{i} C\left(p_{i}, q_{\pi(i)}\right)
$$

which can be computed via the Hungarian algorithm as in Jonker \& Volgenant (1987). To conform to a one-to-one mapping, $|n-m|$ dummy points can be added to the smaller fingerprint minutiae set. Minutiae that are mapped to these dummy points can be considered to be outliers. For more robust handling, dummy point mappings can be extended to minutiae that have a minimum cost greater than a desired threshold $\epsilon_{d}$. 


\subsubsection{Registration using the Thin Plate Spline}

After finding the minutiae correspondences, the Thin Plate Spline (T.P.S) can be used to register the point correspondences together, accounting for a rigid global transformation and local non-linear transformation.

T.P.S is a mathematical model based on algebraically expressing the physical bending energy of a thin metal plate on point constraints. T.P.S is both a simple and sufficient model for non-rigid surface registration with notable applications in medical imaging. T.P.S was first introduced in Bookstein (1989) for the accurate modelling of surfaces that undergo natural warping, where no significant folds or twists occur (i.e., where a diffeomorphism exists).

Two sets of landmark points (i.e. minutiae) from two $\mathbb{R}^{2}$ surfaces are paired in order to provide an interpolation map on $\mathbb{R}^{2} \rightarrow \mathbb{R}^{2}$. T.P.S decomposes the interpolation into a linear component with an affine transformation for a global coarse registration and a non-linear component with smaller non-affine transformations. In other words, the linear component or affine transform can be considered as the transformation that expresses the global geometric dependence of the point sets, whereas the non-affine transform component identifies individual transform components in order to fine tune the interpolation of the point sets. In addition, the affine transform component allows T.P.S to be invariant under both rotation and scale.

In the general two dimensional T.P.S case, we have $n$ control points

$$
\left\{\mathbf{p}_{1}=\left(x_{1}, y_{1}\right), \mathbf{p}_{2}=\left(x_{2}, y_{2}\right), \ldots, \mathbf{p}_{n}=\left(x_{n}, y_{n}\right)\right\}
$$

from an input $\mathbb{R}^{2}$ image and target control points

$$
\left\{\mathbf{p}_{1}^{\prime}=\left(x_{1}^{\prime}, y_{1}^{\prime}\right), \mathbf{p}_{2}^{\prime}=\left(x_{2}^{\prime}, y_{2}^{\prime}\right), \ldots, \mathbf{p}_{n}^{\prime}=\left(x_{n}^{\prime}, y_{n}^{\prime}\right)\right\}
$$

from a target $\mathbb{R}^{2}$ image. To set up the required algebra of the general T.P.S case, we define the following matrices

$$
\mathbf{K}=\left[\begin{array}{cccc}
0 & U\left(r_{12}\right) & \ldots & U\left(r_{1 n}\right) \\
U\left(r_{21}\right) & 0 & \ldots & U\left(r_{2 n}\right) \\
\ldots & \ldots & \ldots & \ldots \\
U\left(r_{n 1}\right) & U\left(r_{n 2}\right) & \ldots & 0
\end{array}\right], n \times n
$$

where $U(r)=r^{2} \log r^{2}$ with $r$ as the Euclidean distance, $r_{i j}=\left\|p_{i}-p_{j}\right\|$,

$$
\begin{gathered}
\mathbf{P}=\left[\begin{array}{ccc}
1 & x_{1} & y_{1} \\
1 & x_{2} & y_{2} \\
\ldots & \ldots & \ldots \\
1 & x_{n} & y_{n}
\end{array}\right], 3 \times n ; \\
\mathbf{V}=\left[\begin{array}{cccc}
x_{1}^{\prime} & x_{2}^{\prime} & \ldots & x_{n}^{\prime} \\
y_{1}^{\prime} & y_{2}^{\prime} & \ldots & y_{n}^{\prime}
\end{array}\right], 2 \times n ; \\
\mathbf{Y}=\left[\begin{array}{ll}
\mathbf{V} & \mathbf{0}_{2 \times 3}
\end{array}\right]^{T},(n+3) \times 2 ;
\end{gathered}
$$

and

$$
\mathbf{L}=\left[\begin{array}{cc}
\mathbf{K} & \mathbf{P} \\
\mathbf{P}^{\mathbf{T}} & \mathbf{0}_{3 \times 3}
\end{array}\right],(n+3) \times(n+3) ;
$$

We can now find the vector $W=\left(w_{1}, w_{2}, \ldots, w_{n}\right)$ and the coefficients $a_{1}, a_{x}, a_{y}$, by the equation 


$$
\mathbf{L}^{-1} \mathbf{Y}=\left(\mathbf{W} \mid a_{1} a_{x} a_{y}\right)^{T}
$$

which can then have its elements used to define the T.P.S interpolation function

$$
f(x, y)=\left[f_{x}(x, y), f_{y}(x, y)\right],
$$

returning the coordinates $\left[x_{\text {res }}, y_{\text {res }}\right]$ compiled from the first column of $\mathbf{L}^{-1} \mathbf{Y}$ giving

$$
f_{x}(x, y)=a_{1, x}+a_{x, x} x+a_{y, x} y+\sum_{i=1}^{n} w_{i, x} U\left(\left\|\mathbf{p}_{i}-(x, y)\right\|\right) .
$$

where $\left[\begin{array}{lll}a_{1, x} & a_{x, x} & a_{y, x}\end{array}\right]^{T}$ is the affine transform component for $x$, and likewise for the second column, where

$$
f_{y}(x, y)=a_{1, y}+a_{x, y} x+a_{y, y} y+\sum_{i=1}^{n} w_{i, y} U\left(\left\|\mathbf{p}_{i}-(x, y)\right\|\right) .
$$

with $\left[a_{1, y} a_{x, y} a_{y, y}\right]^{T}$ as the affine component for $y$. Each point (or minutia location) can now be updated as

$$
\begin{aligned}
& x_{\text {new }}=f_{x}(x, y)=x_{\text {res }} \\
& y_{\text {new }}=f_{y}(x, y)=y_{\text {res }} .
\end{aligned}
$$

It can be shown that the function $f(x, y)$ is the interpolation that minimises

$$
I_{f} \propto \mathbf{W} \mathbf{K} \mathbf{W}^{T}=\mathbf{V}\left(\mathbf{L}_{\mathbf{n}}^{-1} \mathbf{K} \mathbf{L}_{\mathbf{n}}^{-1}\right) \mathbf{V}^{T},
$$

where $I_{f}$ is the bending energy measure

$$
I_{f}=\iint_{\mathbb{R}^{2}}\left(\frac{\partial^{2} z}{\partial x^{2}}\right)^{2}+2\left(\frac{\partial^{2} z}{\partial x \partial y}\right)^{2}+\left(\frac{\partial^{2} z}{\partial y^{2}}\right)^{2} d x d y
$$

and $\mathbf{L}_{\mathbf{n}}$ is the $n \times n$ sub-matrix of $\mathbf{L}$.

Since ill-posed mappings of control points which violate mapping existence, uniqueness, or continuity, can readily exist in real world examples, the use of a Regularization term like Wahba (1990), $\lambda . I_{f}$, can be included in order to smooth the performed interpolation. Thus, the minimization of the error term

$$
H[f]=\sum_{i=1}^{n}\left(v_{i}-f\left(x_{i}, y_{i}\right)\right)^{2}+\lambda . I_{f}
$$

is performed, where the matrix $\mathbf{K}$ is replaced with $\mathbf{K}+\lambda$.I. One should note that $\lambda=0$ results in exact interpolation.

Using the regularized T.P.S transformation method, $n$ iterations are applied, where each iteration has minutiae mappings reassigned and transformation re-estimated using the previous minutiae set transformed state of the test fingerprint (note: the template remains static). 


\subsubsection{Similarity score}

Once the $n$ iterations are performed, the final pairs have now been established. From this, the shape similarity distance measure can be calculated as

$$
D_{s c}(P, Q)=\frac{1}{n} \sum_{p \in P} \arg \min _{q \in Q} C(p, T(q))+\frac{1}{m} \sum_{q \in Q} \arg \min _{p \in P} C(p, T(q))
$$

where $T($.$) denotes the T.P.S transformed representative of the contour point q$. In addition, an appearance term, $D_{a c}(P, Q)$, measuring pixel intensity similarity and a bending energy term, $D_{b e}(P, Q)=I_{f}$, can be added to the similarity score.

Afterward, the similarity measure was modified as

$$
D_{s c}^{*}=D_{s c}+\beta D_{b e}
$$

Although this measure does not take into account the strict one-to-one mapping of minutiae; through experimentation, this method proved to be sound, providing acceptable performance in fingerprint similarity assessment. However, the resulting minutiae mapping from the application of the Hungarian algorithm on the contextually based cost histograms produced some un-natural pairs, as illustrated in Figure 8 (top). This is due to the lack of a minutiae pair pruning procedure. The existence of un-natural pairs could potentially skew the Thin Plate Spline (T.P.S) linear transform performed. In addition, such pairs generally increase the bending energy substantially, thus leading to invalid matching results, particularly for genuine matches.

\subsection{Proposed matching method}

Recently, hybrid matching algorithms have been used for fingerprint matching. Although minutiae detail alone can produce a highly discriminant set of information, the combination of level 1 feature, such as orientation and frequency, and other level features, can increase discriminant information, and hence, increase matching accuracy, as illustrated in Benhammadi et al. (2007), Youssif et al. (2007), Reisman et al. (2002), and Qi et al. (2004).

The detailing of the proposed hybrid matching algorithm based on a modified version of the enhanced shape context method in Kwan et al. (2006), along with the integration of the orientation-based descriptor of Tico \& Kuosmanen (2003) is given here, illustrating a significant performance improvement over the enhanced shape context method of Kwan et al. (2006). The main objective of the integration is two-fold, firstly to prune outlier minutiae pairs, and secondly to provide more information to use in similarity assessment.

As briefly described earlier in section 2.3, the orientation-based descriptor in Tico \& Kuosmanen (2003) utilises the orientation image to provided local samples of orientation around minutiae in a concentric layout. Each orientation sample point is calculated as

$$
\theta_{c, d}^{A_{i}}=\min \left(\left|\theta_{c, d}^{s_{A}}-\theta_{A_{i}}\right|, \pi-\left|\theta_{c, d}^{s_{A}}-\theta_{A_{i}}\right|\right)
$$

being the $d^{\text {th }}$ sample on the $c^{\text {th }}$ concentric circle with distance $r_{c}$ away from the minutiae point $m_{A_{i}}$, where $\theta_{A_{i}}$ and $\theta_{c, d}^{s_{A}}$ are the minutia and sample point orientation estimations, respectively. The orientation distance of equation 22 is used to prune outlier pairs resulting from the Hungarian algorithm which produced the mapping permutation of equation 30 . Although the shape context defines the similarity measure in equation 47-48 with no strict one-to-one correspondence, minutiae should have a more strict assessment based on the optimal mapping, since minutiae are key landmarks as opposed to randomly sampled contour 

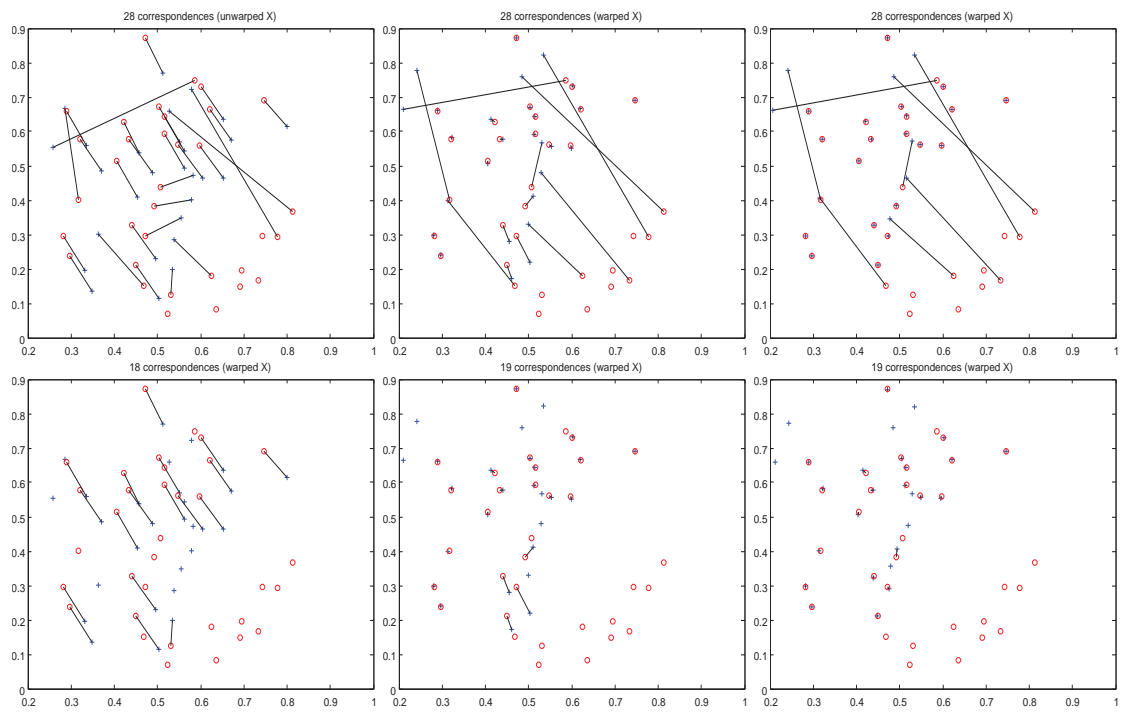

Fig. 8. top: The Enhanced Shape Context method producing initial minutiae pair correspondences. The next two images are for the following iterations. One should note that there are some clear un-natural pairs produced, where plate foldings are evident (i.e. pair correspondences that cross other pair correspondences). bottom: The proposed hybrid method initial minutiae pair correspondences along with following iterations of produced correspondences.

points. Thus, equation 48 can be modified to only score the pairs with the optimal one-to-one mapping as

$$
D_{s c}^{* *}(P, Q)=\frac{1}{n} \sum_{p_{i} \in P \mid D_{o}\left(p_{i}, q_{\pi(i)}\right)<\delta} C\left(p_{i}, q_{\pi(i)}\right)+\Lambda D_{o}\left(p_{i}, q_{\pi(i)}\right)+\beta D_{b e}
$$

with an addition term in the summation to account for orientation distance scaled by the tunable parameter, $\Lambda$ with range $[0,1]$.

In terms of what concentric circle radii and sample configuration should be used, the method explained in Tico \& Kuosmanen (2003) prescribes that the radius for circle $K_{l}$ be $r_{l}=2 l \times \tau$, where $\tau$ denotes the average ridge period, and for the sample configuration, the circle $K_{l}$ should have roughly $\left\lceil\frac{\pi r_{1}}{\tau}\right\rceil$. As the average ridge period was recorded to be $0.463 \mathrm{~mm}$ in Stoney (1988), for a fingerprint image with dots per inch (dpi) equal to $R$, the previous formula for the configuration can be expressed as $K_{l}=\left\lceil\frac{172 . r_{l}}{R}\right\rceil$. However, this was really only used as a rough guide for the configuration used in the experimentation of Tico \& Kuosmanen (2003). This was also used as a rough guide for our implementation of the orientation-based descriptor.

Although the log-polar space adequately provides extra importance toward neighbouring sample points, additional emphasis on local points may be desirable, since minutiae sets are largely incomplete and do not entirely overlap. For a given minutia, non-local bin regions may be partially or largely outside the segmented region of interest for one fingerprint and not the other. In addition, the non-local bins are spatially larger, and hence, have a higher probability 
of containing false minutia caused by noises. Such issues can potentially result in incorrect minutiae pairs. Thus, the enhanced shape context's log-polar histogram cost in equation 27 is adjusted to contain a tunable Gaussian weighting of histogram bin totals, depending on their distances away from the centre (reference minutia), with

$$
C^{* *}\left(p_{i}, q_{j}\right)=\left(1-\gamma C_{i j}^{\text {type }} C_{i j}^{\text {angle }}\right) \cdot\left(\frac{1}{2} \sum_{k=1}^{K} \frac{\left[h_{p_{i}}(k)-h_{q_{j}}(k)\right]^{2}}{h_{p_{i}}(k)+h_{q_{j}}(k)} \times \exp \left(-\frac{\left(r_{k}-r_{\text {min }}\right)^{2}}{2 \sigma^{2}}\right)\right)
$$

where $r_{\min }$ is the outer boundary of the closest bin, $r_{k}$ is the current bin outer boundary distance, and $\sigma^{2}$ is a tunable parameter (see Figure 9).

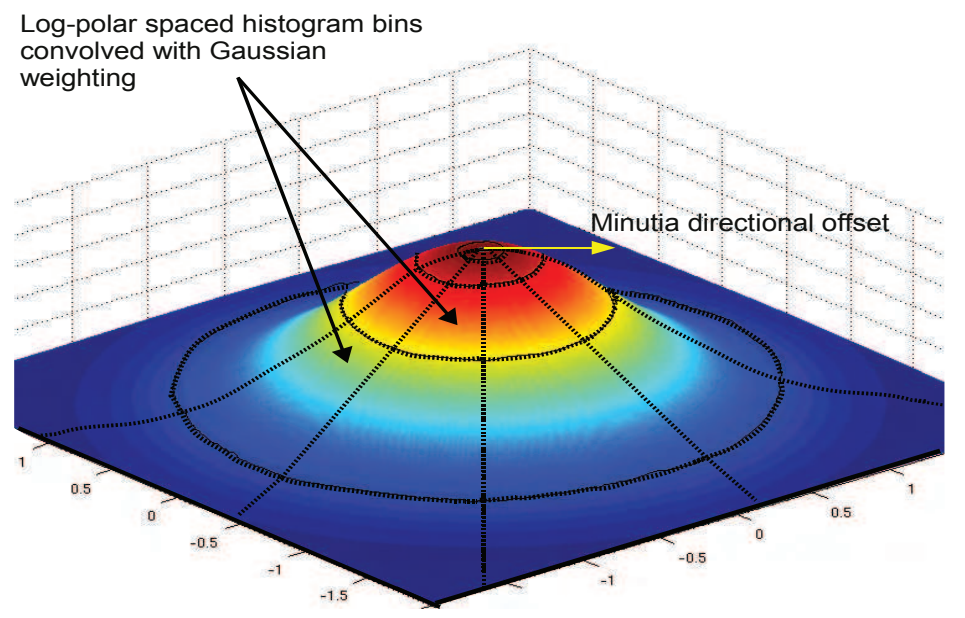

Fig. 9. The log-polar sample space convolved with a two dimensional Gaussian kernel, resulting in each bin to be weighted according to its distance from the origin (minutia). The histogram cost calculation uses the direction of the reference minutia as the directional offset for the bin order, making the descriptor invariant to rotation.

\subsubsection{Adaptive greedy registration}

If we re-examine the registration of the enhanced shape context method of Kwan et al. (2006) discussed in section 3.1, we can summarise the iterative process as updating minutiae pairs with the enhanced shape context descriptor, followed by using T.P.S to perform a global alignment with the linear transform component, and then performing non-linear transform to model warping caused by skin elasticity. This method does not utilise any singularities for the registration process, solely relying on the T.P.S framework for registration. Hence, registration is largely reliant on the accuracy of the spatial distribution of minutiae relative to a given reference minutia, which is often inadequate.

The proposed method does not use the T.P.S transform to perform the initial affine transform. Instead, a greedy method similar to Tico \& Kuosmanen (2003) (of equations 20-23) is used. 
Using the minutiae set representation of equations 3 and 4 , each possible pair $\left(m_{A_{i}}, m_{B_{j}}\right)$ of the affine transform, $T$, is calculated by using the orientation differences of each minutiae direction as the rotation component, and the difference in $x-y$ coordinates as the offset component (as illustrated in equations 7-9). The heuristic that requires maximising is

$$
H(A, B)=\arg \max _{\psi} \frac{\sum_{i} S\left(m_{A_{i}}, m_{B_{T(i)}}\right)}{n\left(\Phi_{\psi}\right)}
$$

where $S($.$) is previously defined in equation 22$ as the similarity function based on the orientation-based descriptor, $\psi$ is the index pair reference for the transform $T$ (i.e. $(i, j)$ with $\left.m_{A_{i}}=m_{B_{T(j)}}\right), \Phi_{\psi}$ is the anchor point set (with size $n\left(\Phi_{\psi}\right)$ ) of minutiae pairs $\left(m_{A_{p}}, m_{B_{q}}\right) \in \Phi_{\psi}$ which have each other as closest points from the opposite minutiae sets with distance less than an empirically set limit, $\delta_{M}$, after applying the given transform. In addition, all anchor point minutiae pairs must have similar orientation, hence meeting the constraint

$$
\min \left(\left|\theta_{A_{i}}-\theta_{B_{j}}\right|, \pi-\left|\theta_{A_{i}}-\theta_{B_{j}}\right|\right)<\delta_{\theta}
$$

(see Figure 5 left). From the given definition, we can easily verify that $(i, j) \in \Phi_{\psi}$.

If the primary core point exists in both the test and template feature sets, provided that the core point detection is highly accurate with a given test dataset, then we have good reason to ignore affine transforms that cause the core points to be greater than a fixed distance, $\delta_{D}$, away from each other. Additional pruning can be achieved by only allowing transforms where the reference minutiae pair has a orientation-based descriptor similarity score to meet

$$
S\left(p_{i}, q_{\pi(i)}\right)<\delta_{S}
$$

where $\delta_{S}$ is empirically set. Such restrictions will not only improve performance, but also make the registration process much more accurate, since we are not just blindly maximising the heuristic of equation 52.

Many registration algorithms use singularities, texture, and minutiae pair information as tools for finding the most likely alignment. However, the overlapped region shape similarity retrieved from minutiae spatial distribution information provides an additional important criteria. After finding the bounding box (overlapping region) of a possible affine transform meeting all prior restrictions, we can then measure shape dissimilarity via the application of the shape context to all interior points $P \subseteq A$ and $Q \subseteq B$ with equation 50, giving additional criteria for affine transforms to meet $D_{s c}(P, Q)<\delta_{s c}$ for an empirically set threshold, $\delta_{s c}$.

For a candidate transform, we have the corresponding anchor point set $\Phi_{\psi}$. We will now define two additional nearest neighbour sets of the interior points in the overlap region (as depicted in Figure 10) as

$$
\Phi_{\alpha}=\left\{i \mid \sqrt{\left(x_{A_{i}}-x_{B_{j}}\right)^{2}+\left(y_{A_{i}}-y_{B_{j}}\right)^{2}}<\delta_{N}\right\}
$$

where $\Phi_{\alpha}$ contains all interior nearest neighbour indices from fingerprint $A$, and likewise, $\Phi_{\beta}$, containing interior nearest neighbour indices for fingerprint $B$. Thus, we have

$$
\Phi_{\psi} \subseteq\left\{(i, j) \mid i \in \Phi_{\alpha} \text { and } j \in \Phi_{\beta}\right\} .
$$

with $1 \leq i \leq p$ and $1 \leq j \leq q$. The affine transform method is detailed in algorithm 1 .

If a candidate affine transform meets the heuristic of equation 52 with the given constraints, 


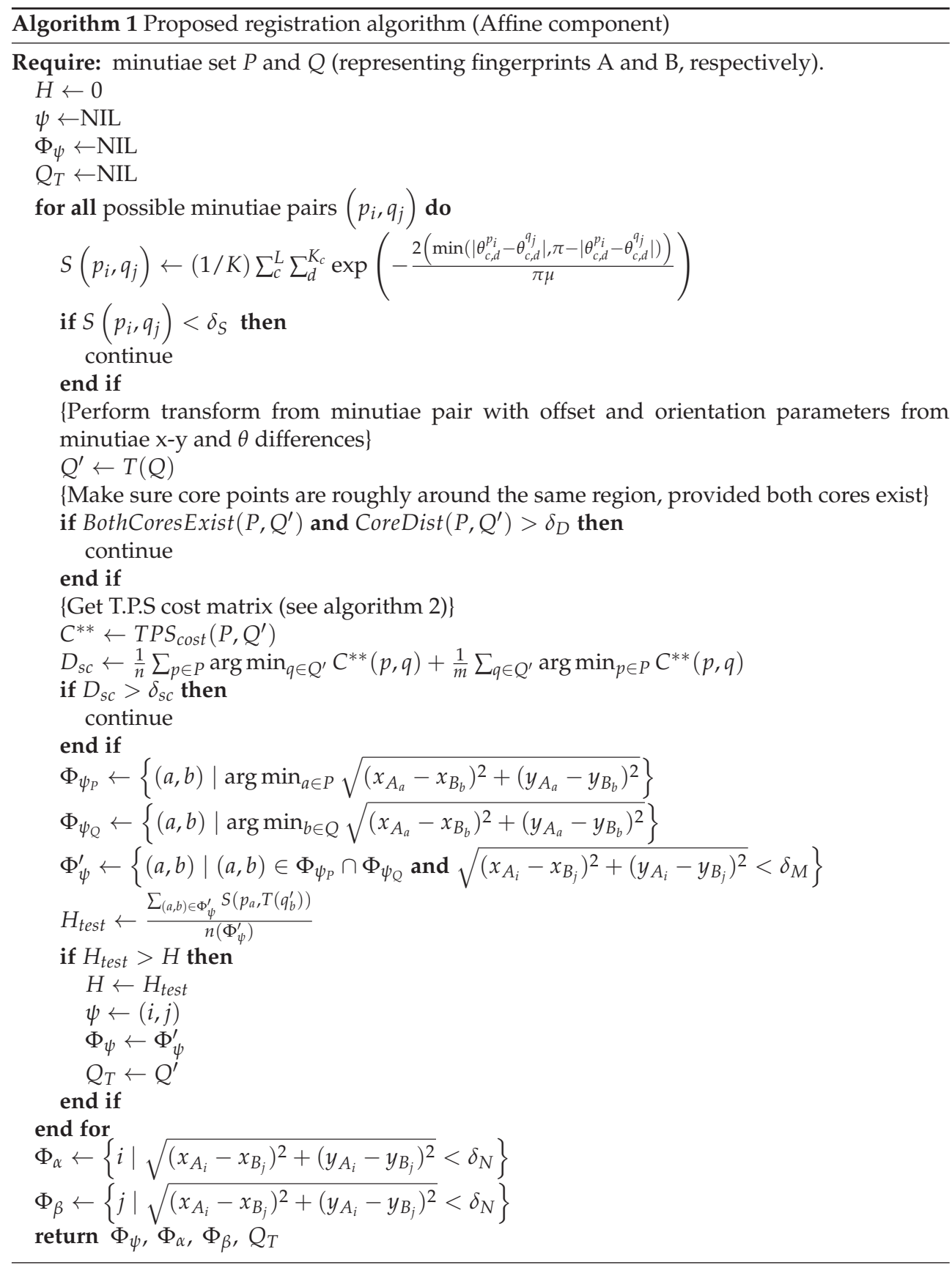




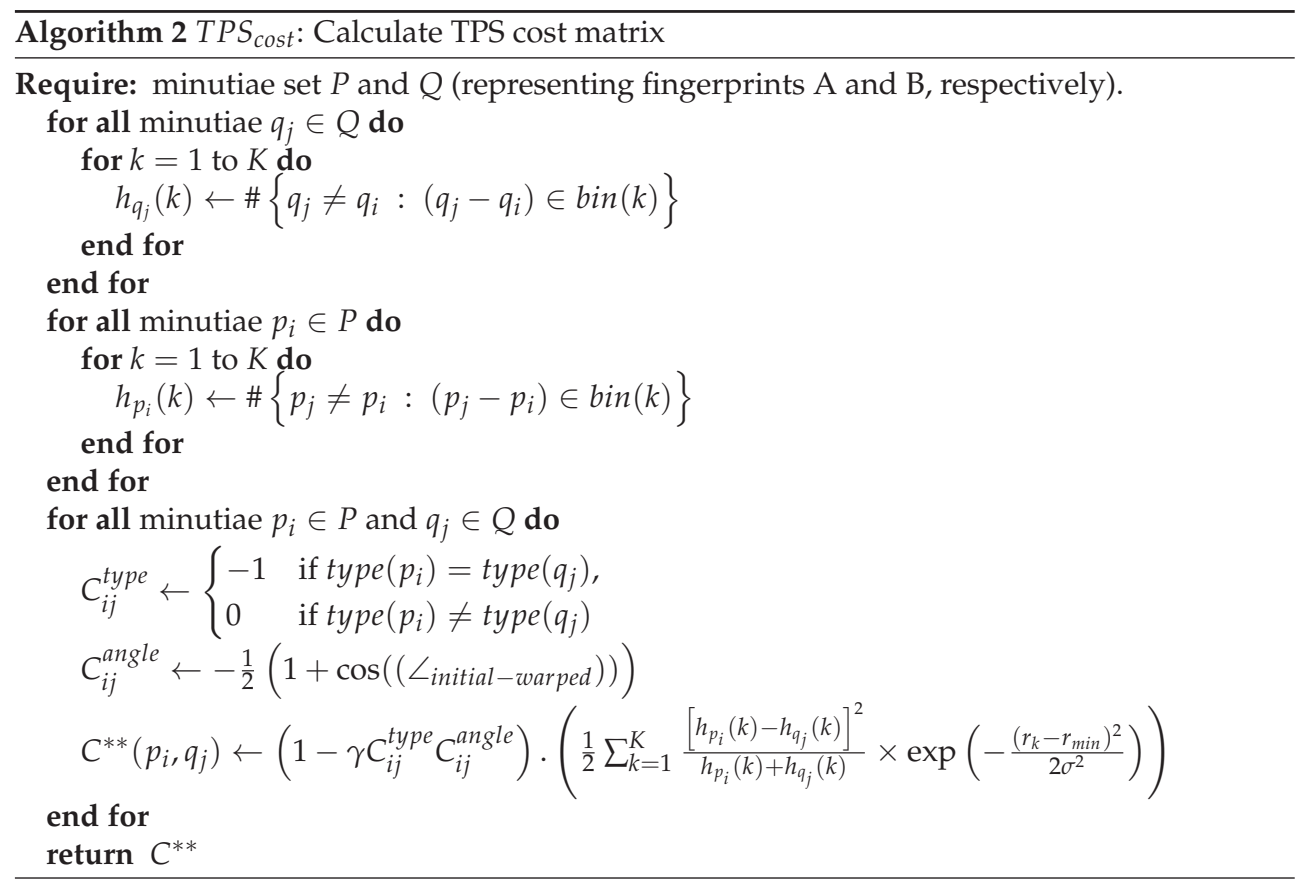

we can then focus on the non-affine aspect of registration. The T.P.S non-affine component adequately modeled the warping caused by skin elasticity in the previously proposed method. With this in mind, it will be desirable to utilise the non-linear component.

The T.P.S has its point correspondence method modified through having anchor point correspondences remain static throughout the iterative process, thus attempting to restrict the affine transform component of T.P.S while finding new correspondences from $\Phi_{\alpha}$ on to $\Phi_{\beta}$ that do not exist in $\Phi_{\psi}$. The shape context's log-polar histogram cost is modified from equation 51 as

$$
C^{\gamma}\left(p_{i}, q_{j}\right)=\gamma_{d} \gamma_{\theta} C^{* *}\left(p_{i}, q_{j}\right)
$$

where $\gamma_{d}$ is defined as

$$
\gamma_{d}= \begin{cases}1 & \text { if } \operatorname{dist}\left(p_{i}, q_{j}\right)<\delta_{\max } \\ \infty & \text { otherwise }\end{cases}
$$

with $\delta_{\max }$ set as the maximum feasible distance caused by warping after applying the candidate affine transform, and similarly, $\gamma_{\theta}$, is defined as

$$
\gamma_{\theta}= \begin{cases}1 & \text { if } \min \left(\left|\theta_{A_{i}}-\theta_{B_{j}}\right|, \pi-\left|\theta_{A_{i}}-\theta_{B_{j}}\right|\right)<\theta_{\text {max }} \\ \infty & \text { otherwise }\end{cases}
$$

with $\theta_{\max }$ set as the maximum feasible orientation difference caused by orientation estimation error in the extraction process. 


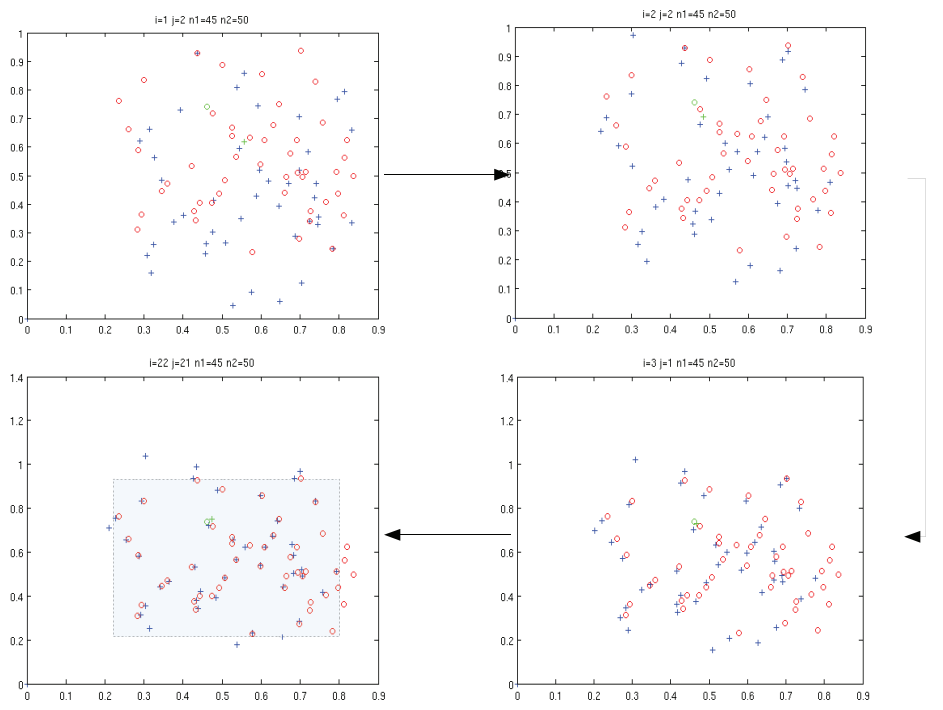

Fig. 10. An example affine transform candidate search sequence with final state and corresponding bounding box for the overlapped region.

$>$ From the new cost function, the Hungarian algorithm is then used to find additional one-to-one correspondences to what already existed in the anchor point set. The candidate affine transform in comparison to the affine component of T.P.S,

$$
\mathbf{A}_{\mathbf{T}}=\left[\begin{array}{ccc}
a_{x, x} & a_{x, y} & a_{1, x} \\
a_{y, x} & a_{y, y} & a_{1, y} \\
0 & 0 & 1
\end{array}\right],
$$

is used to assess the accuracy of the additional correspondences found. The T.P.S affine component would not have prominent translation, rotation, and shear parameters since the candidate transform should have already adequately dealt with the affine registration task as the Euclidean constraints on the anchor point worked to keep the global transform rigid. In addition, the inclusion of additional natural minutiae correspondences should not significantly alter the affine registration required. Thus, the lack of prominent translation, rotation, and shear parameters for the T.P.S affine transform indicates an existing agreement between both affine transforms. Evaluation of the translation distance is given by

$$
r_{\text {affine }}=\sqrt{a_{1, x}^{2}+a_{1, y}^{2}}<r_{\max } .
$$

Using the Singular Value Decomposition $(S V D)$ of the non-translation components of $\mathbf{A}_{\mathbf{T}}$ :

$$
\operatorname{SVD}\left(\left[\begin{array}{ll}
a_{x, x} & a_{x, y} \\
a_{y, x} & a_{y, y}
\end{array}\right]\right)=U D V^{T}
$$

where $U, V^{T} \in S O(2, \mathbb{R})$ (i.e. $2 \times 2$ dimension rotation matrices with angles $\theta_{\alpha}$ and $\theta_{\beta}$, respectively) and $D$ is a $2 \times 2$ diagonal matrix representing scaling along the rotated coordinate 


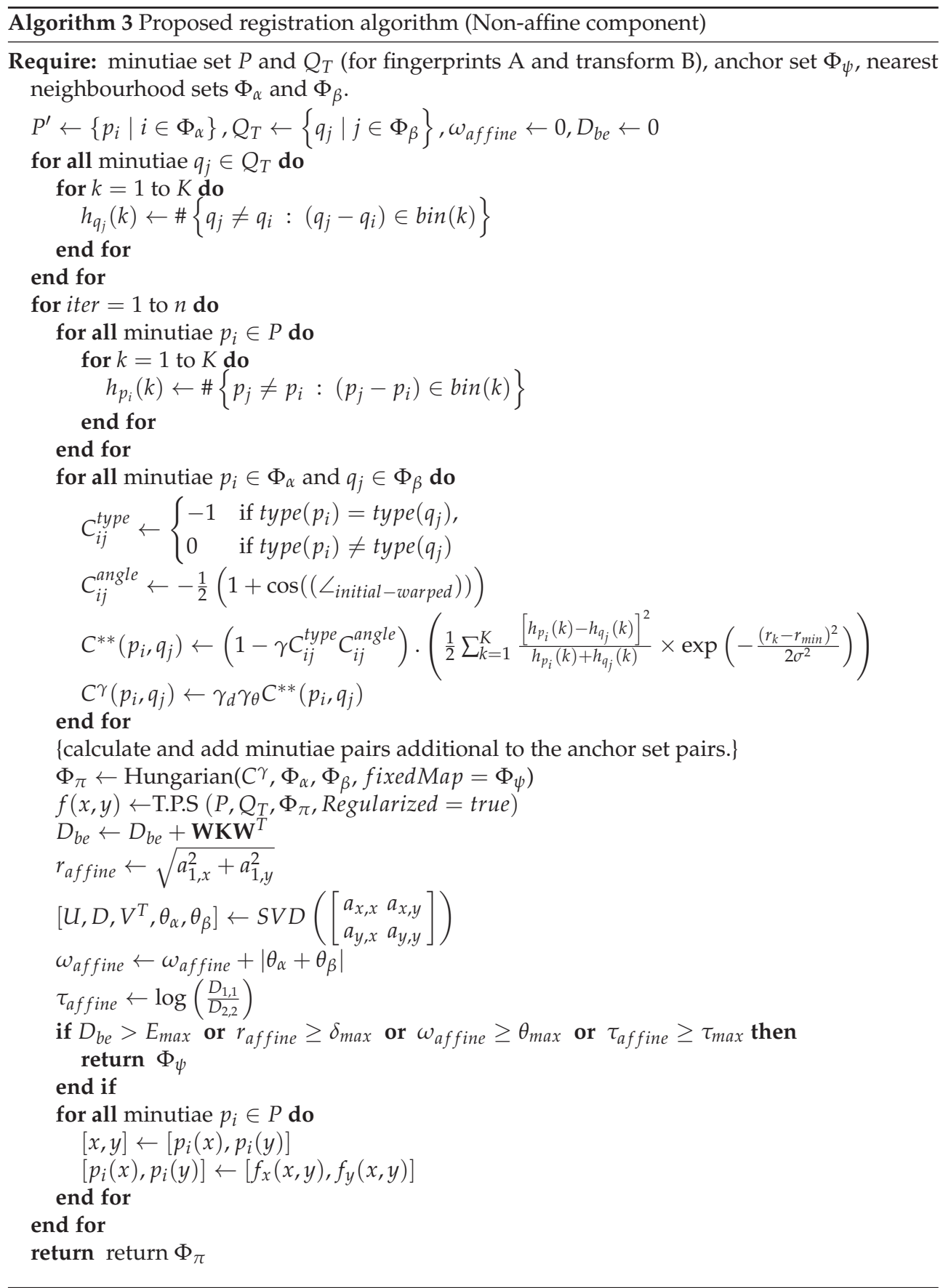


axes of $V^{T}$ with nonnegative diagonal elements in decreasing order, the evaluation of rotation

$$
\omega_{\text {affine }}=\left|\theta_{\alpha}+\theta_{\beta}\right|<\omega_{\max }
$$

and shear

$$
\tau_{\text {affine }}=\log \left(\frac{D_{1,1}}{D_{2,2}}\right)<\tau_{\text {max }}
$$

is performed for empirically set values $\omega_{\max }$ and $\tau_{\max }$. If the above affine transform criteria of equations 61, 63, and 64 are not met, no extra minutiae pairs are produced. Unlike the previous method, this helps uphold spatial consistency by not creating un-natural pairs (see Figure 8 (bottom)). Essentially, the candidate affine transform is used as the ground truth registration over the T.P.S affine component.

A final integrity check of the validity of the additional minutiae pairs produced from the non-affine transform is the measured bending energy, previously defined in equation 45 . If the non-affine transform produces a bending energy distance $D_{b e}>E_{\text {max }}$, then all additional minutiae pairs are also rejected, in order to avoid un-natural warping to occur. The non-affine transform component is detailed in algorithm 3.

\subsubsection{Matching algorithm}

Once the minutiae pairs have been established, pruning is performed to remove unnatural pairings. However, if we closely analyse the orientation-based descriptor used for pruning, we can see that a fundamental flaw arises with partial fingerprint coverage, specifically for minutiae pairs near fingerprint image edges. In such a case, the typical formula for distance calculation cannot count orientation samples that lie outside the region of interest, and therefore, unnecessarily reduces the orientation distance measure (see Figure 11). Moreover, regions that have high noise also cannot have their orientation reliably estimated due to information to be missing (if regions with high noise are masked), and likewise, reduces the orientation-based descriptor common region coverage.

A proposed modification to the orientation-based descriptor is applied so that the amount of common region coverage that each descriptor has is reflected in the similarity score. This is achieved by a simple Gaussian weighting of equation 22 with

$$
S^{*}\left(m_{A_{i}}, m_{B_{j}}\right)=S\left(m_{A_{i}}, m_{B_{j}}\right) \times \exp \left(-\max \left(0, \Delta_{\text {cutoff }}-\Delta_{g_{-} \text {count }}\right) \cdot \mu_{S}\right)
$$

where $\Delta_{\text {cutoff }}$ is the cutoff point where all good sample totals below this value are weighed, $\Delta_{g_{-} c o u n t}$ is the total number of good samples (i.e. where a good sample is defined to be in a coherent fingerprint region), and $\mu_{s}$ is a tunable parameter. However, for a more exhaustive approach, one could empirically review the estimated distribution of orientation-based similarity scores for true and false cases, with specific attention towards the effect of coverage completeness on the accuracy of the similarity measure.

Equation 65 relies on the intersection set of valid samples for each minutiae, defined as

$$
I\left(A_{i}, B_{j}\right)=\left\{s \mid s \in\left\{L, K_{c}\right\} \text { and } \operatorname{valid}\left(A\left(s_{x}, s_{y}\right)\right)\right\} \cap\left\{t \mid t \in\left\{L, K_{c}\right\} \text { and } \operatorname{valid}\left(B\left(t_{x}, t_{y}\right)\right)\right\}
$$

where $L$ is the sample position set and $K_{c}$ is the concentric circle set. Thus, we can also define a variant of the function $S^{*}\left(m_{A_{i}}, m_{B_{j}}, I\right)$ where a predefined sample index set, $I$, is given to indicate which samples are only to be used for the similarity calculation, ignoring $i \notin I$ even if corresponding orientation samples are legitimately defined for both fingerprints (note: this variant is used later for similarity scoring in the matching algorithm). 

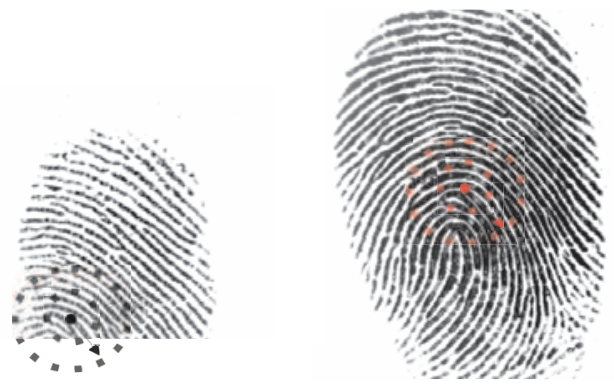

Fig. 11. An example where the orientation-based descriptor for corresponding minutiae in two different impressions of the same fingerprint have no coverage in a substantial portion of the orientation sample.

After the filtered minutiae pair set is produced, we can now assess the similarity of the pairs. Minutiae $\delta$-neighbourhood structure families, where particular spatial and minutiae information of the $\delta$ closest minutiae to a reference minutiae are extracted as features, have been used before in minutiae based matching algorithms (such as Chikkerur \& Govindaraju (2006) and Kwon et al. (2006)) for both alignment and similarity measure (see Figure 12). The $\delta$-neighbourhood structure proposed has the following fields:

- Distance $d_{i}$ : the distance a neighbourhood minutia is away from the reference minutia.

- Angle $\angle_{i}$ : the angle a neighbourhood minutia is from the reference minutia direction.

- Orientation $\theta_{i}$ : the orientation difference between a neighbourhood minutia direction and the reference minutia direction.

- Texture $\Gamma_{i}$ : the orientation-based descriptor sample set for a neighbourhood minutia used to measure the region orientation similarity with the reference minutia for a given sample index.

giving us the sorted set structure

$$
n \delta\left(m_{A_{i}}\right)=\left\{\left\{d_{A_{i}(1)}, \angle_{A_{i}(1)}, \theta_{A_{i}(1)},\left\{\Gamma_{A_{i}(1)}\right\}\right\}, \ldots,\left\{d_{A_{i}(\delta)}, \angle_{A_{i}(\delta)}, \theta_{A_{i}(\delta)},\left\{\Gamma_{A_{i}(\delta)}\right\}\right\}\right\}
$$

being sorted by the distance field in ascending order. The first two fields are considered as the polar co-ordinates of the neighbourhood minutiae with the referencing minutia as the origin, and along with the third field, they are commonly used in local neighbourhood minutiae based structures ( Kwon et al. (2006) and Jiang \& Yau (2000)). Using the modified weighted orientation-based descriptor, the texture field provides additional information on how each local orientation information surrounding the $\delta$-neighbourhood minutiae set vary from that of the reference minutia, with the measure rated using equation 65 . These structures can now be used to further assess and score the candidate minutiae pairs.

When comparing the $\delta$-neighbourhood elements of a candidate minutiae pair, the first three fields are straight forward to compute the differences with 


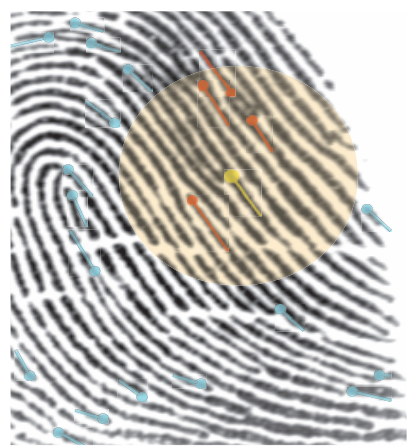

Fig. 12. The $\delta$-neighbourhood ( $\delta=4)$ for a given minutia (ignoring false crease minutiae).

$$
\begin{gathered}
r_{\operatorname{diff}\left(m_{A_{i}}, m_{B_{j}}\right)}(x, y)=\left|d_{A_{i}(x)}-d_{B_{j}(y)}\right|, \\
\angle_{\operatorname{diff}\left(m_{A_{i}}, m_{B_{j}}\right)}(x, y)=\min \left(\left|\angle_{A_{i}(x)}-\angle_{B_{j}(y)}\right|, 2 \pi-\left|\angle_{A_{i}(x)}-\angle_{B_{j}(y)}\right|\right), \\
\theta_{\operatorname{diff}\left(m_{A_{i}}, m_{B_{j}}\right)}(x, y)=\min \left(\left|\theta_{A_{i}(x)}-\theta_{B_{j}(y)}\right|, \pi-\left|\theta_{A_{i}(x)}-\theta_{B_{j}(y)}\right|\right),
\end{gathered}
$$

However, to make an accurate comparison of a minutiae pair's $\delta$-neighbourhood orientation similarity information, we must find the intersection of sample positions that are within valid and coherent regions for both images (see Figure 13). Thus, the fourth field is composed of the set of all orientation samples and is used to dynamically calculate the orientation difference of neighbouring minutiae for a minutiae pair's respective $\delta$-neighbourhoods, using

$$
\Gamma_{\operatorname{diff}\left(m_{A_{i}}, m_{B_{j}}\right)}(x, y)=\left|\Gamma_{A_{i}(x)}-\Gamma_{B_{j}(y)}\right|
$$

where $\Gamma_{A_{i}(x)}=S^{*}\left(m_{A_{i}}, m_{A_{i}(x)}, I_{0}\right), \Gamma_{B_{j}(y)}=S^{*}\left(m_{B_{j}}, m_{B_{j}(y)}, I_{0}\right)$, and overlap index set $I_{0}=$ $I\left(A_{i}, A_{i}(x)\right) \cap I\left(B_{j}, B_{j}(y)\right)$.

We now require a systematic method for scoring a minutiae pair given their corresponding $\delta$-neighbourhoods. Since there is no guarantee that each $\delta$-neighbourhood has the same minutiae set, optimal mapping methods, such as the Hungarian algorithm, may not be desirable. Instead, we use a novel greedy algorithm, where we iterate through one neighbourhood and find the best match from another, provided that they meet pre-defined affine constraints of equations 61-63. Removal of the matching elements from the $\delta$-neighbourhood lists ensures one-to-one mappings of $\delta$-neighbourhood minutiae.

A $\delta$-neighbourhood similarity score is tallied for the candidate minutiae pair $\left(m_{A_{i}}, m_{B_{j}}\right)$ for each matched $\delta$-neighbourhood minutiae pair having respective neighbourhood sorted set indexes $\left(x_{s}, y_{t}\right)$, found to also match, using

$$
\operatorname{sim}_{\delta}\left(m_{A_{i}}, m_{B_{j}}\right)=\sum_{s, t}\left(\alpha\left(x_{s}, y_{t}\right)+\gamma \beta\left(x_{s}, y_{t}\right)\right)
$$

where

$$
\alpha(x, y)=\exp \left(-r_{\operatorname{diff}\left(m_{A_{i}}, m_{B_{j}}\right)}(x, y)-\theta_{\operatorname{diff}\left(m_{A_{i}}, m_{B_{j}}\right)}(x, y)-\angle_{\operatorname{diff}\left(m_{A_{i}}, m_{B_{j}}\right)}(x, y)\right)
$$




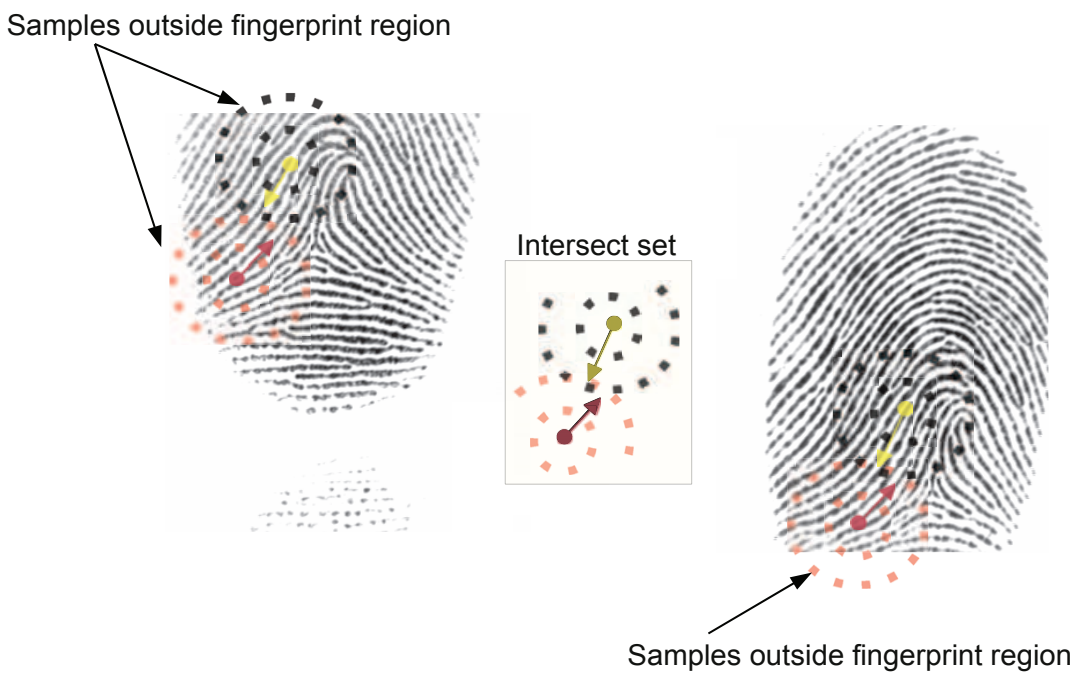

Fig. 13. An example where the orientation-based descriptor for corresponding minutiae in two different impressions of the same fingerprint have no coverage in a substantial portion of the orientation sample.

and

$$
\beta(x, y)=\exp \left(-\Gamma_{\operatorname{diff}\left(m_{A_{i}}, m_{B_{j}}\right)}(x, y)\right)
$$

with a tunable parameter $\gamma$ defined in $[0,1]$.

After all candidate minutiae pair $\delta$-neighbourhoods have been scored, we can now find a fingerprint matching similarity score as

$$
\operatorname{sim}(A, B)=\frac{n_{M}\left(\sum_{(i, j)} \operatorname{sim}_{\delta}\left(m_{A_{i}}, m_{B_{j}}\right)\right) \cdot\left(\sqrt{S_{\max }}\right)}{n_{A} \cdot n_{B}}-v D_{s c}^{* *}
$$

where

$$
S_{\text {max }}=\arg \max _{(i, j)} S^{*}\left(m_{A_{i}}, m_{B_{j}}, I\left(A_{i}, B_{j}\right)\right)
$$

with $S^{*}\left(m_{A_{i}}, m_{B_{j}}, I\left(A_{i}, B_{j}\right)\right)$ defined in equation 65 as the orientation-based descriptor similarity measure, $D_{S c}^{* *}$ is the modified shape context distance in equation $50, n_{M}$ is the number of matching filtered minutiae pairs, $n_{A}$ and $n_{B}$ are the number of minutiae in the overlap region from fingerprint $A$ and $B$, respectively, $i$ and $j$ are the index of the filtered minutiae pair elements in fingerprint $\mathrm{A}$ and $\mathrm{B}$, respectively, and $v$ a tunable parameter in $[0,1]$. Additionally, we can add the type similarity of each pair to equation 71 by adding a small constant, $\zeta_{t}$, when the minutiae types agree. The matching method is summarised in algorithm 4 . 


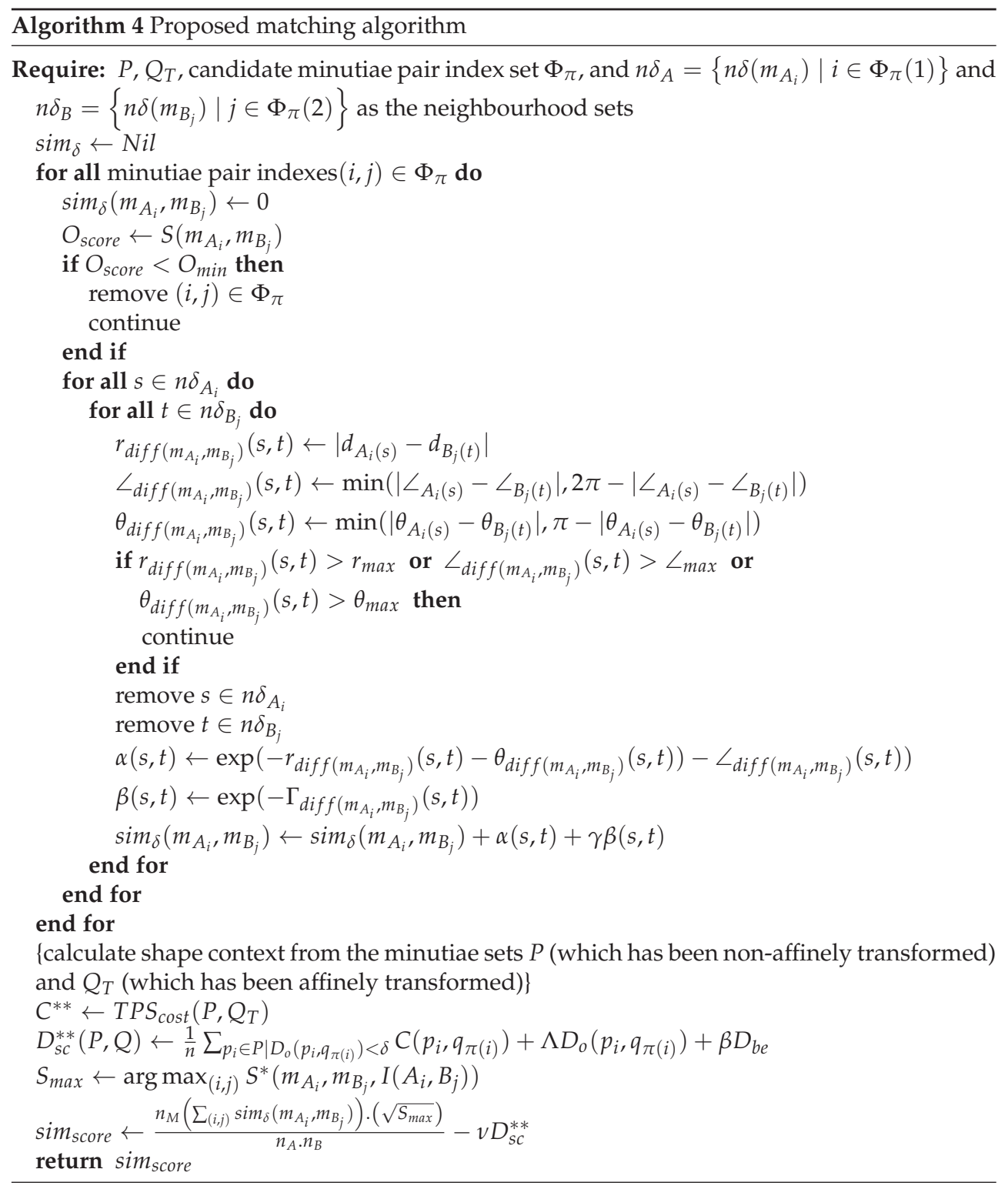




\section{Experimental results}

The experiment was performed on two databases, the FVC2002 database Db1 set A ( Maio et al. (2002)) which contains 800 fingerprint images with 100 fingers having 8 impressions each, and fingerprint database from the University of Bologna ( Bologna (2000)), consisting of 168 fingerprint images formed by 21 fingers with 8 impressions each. The parameters of the algorithm (see Table 1) were tuned with the FVC2002 database Db1 set B, which contains 80 fingerprints (10 fingers with 8 impressions each). The program was written in Matlab and run on a 1.66GHz Linux PC with 2Gb memory. The FVC2002 protocol ( Maio et al. (2002)) was used in experimentation, comprising of $n \times 8 \times 7 / 2=2800$ genuine and $n \times(n-1) / 2=4950$ imposter attempts for the FVC2002 database, and $n \times 8 \times 7 / 2=588$ genuine and $n \times(n-$ 1) $/ 2=210$ imposter attempts for the smaller database.

Image enhancement for the FVC2002 database was performed via the STFT method ( Chikkerur et al. (2004)), while the binarization/thinning based minutiae extraction method and smoothed orientation image creation proposed in Hong et al. (1998) were used for feature extraction, along with the core point detection algorithm based on the method described in Julasayvake \& Choomchuay (2007). Spurious minutiae had very crude filtering applied, with only short spurs and minutiae near segmented border regions removed. All other false minutia structures remained in the feature set. In addition, the thinning algorithm is known to produce a higher number of spurious minutia in comparison to ridge following methods. Moreover, the STFT method was noted in Jirachaweng et al. (2009) to produce a high rate of spurious minutiae, while the extraction method encountered a substantial amount of minutia type interchange (approx. 30\%). Thus, we can expect our feature set to have at least a moderate amount of noise.

Table 2 summarises the performance of our algorithm against numerous well known algorithms on the FVC2002 database, as does Table 3 with the University of Bologna database. One should note that the parameters were not tuned for this second database, but still managed to perform quite well. Figures 14 and 15 show the FMR vs. FNMR graphs. The proposed method managed to finish in a top 8 position for the FVC2002 database. Figure 16 illustrates the genuine and imposter distributions of the similarity score for both databases.
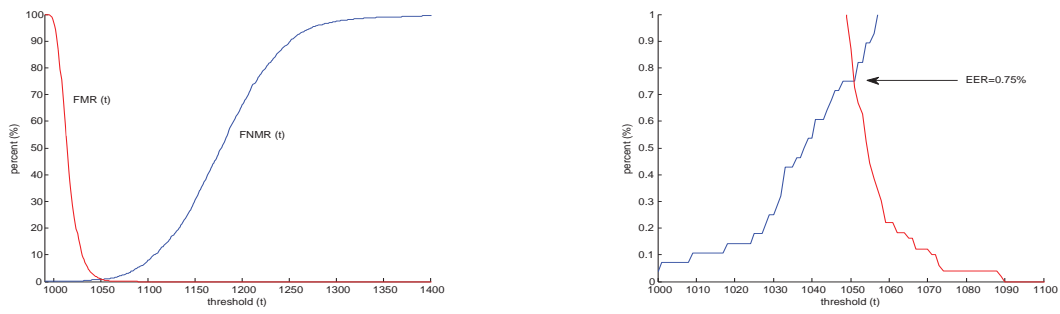

Fig. 14. left: Proposed method FNMR and FMR vs matching threshold $t$ on FVC2002 Db1 Set A right: Close up illustrating the EER (0.75\%).

\section{Conclusions}

The proposed fingerprint matching method using a hybrid shape and orientation descriptor outperforms many well-known methods on the FVC2002 database (in the top 8th place) in the 


\begin{tabular}{|l|c|c|c|}
\hline Parameter Name & Hybrid Component & Relevance & Value \\
\hline iterations & shape context & T.P.S & 5 \\
annealing rate & shape context & T.P.S & 0.35 \\
regularization & shape context & T.P.S & 0.8 \\
inner/outer radii & shape context & log-polar & $1 / 64,2$ \\
radii/theta bins & shape context & log-polar & 8,10 \\
$\sigma^{2}$ & shape context & log-polar & 4.5 \\
circle radii $r_{l}$ & orientation & minutia & $7,14,21,28,35,42,49$ \\
circle samples & orientation & minutia & $12,16,22,28,32,36,36$ \\
$\nu$ & scoring & shape context & 0.3 \\
$O_{\min }$ & matching & orientation & 0.25 \\
$\delta_{\max }$ & registration & T.P.S & 0.1 \\
$\delta_{S}$ & registration & orientation & 0.25 \\
$\delta_{D}$ & registration & core & 30 pixels \\
$\delta_{\text {Sc }}$ & registration & shape context & 4.9 \\
$r_{\max }$ & registration & T.P.S & 0.1 \\
$\omega_{\max }$ & registration & T.P.S & $\pi / 6$ \\
$\tau_{\max }$ & registration & T.P.S & 0.3 \\
$E_{\max }$ & registration & T.P.S & 12 \\
$\Delta_{\text {cutoff }}$ & matching & filtering & 70 \\
$L_{\max }$ & matching & T.P.S & $\pi / 8$ \\
$r_{\max }$ & matching & T.P.S & 0.05 \\
$\theta_{\max }$ & matching & T.P.S & $\pi / 8$ \\
$\delta$ & matching & neighbourhood & 4 \\
\hline
\end{tabular}

Table 1. Parameters setup for experimentation
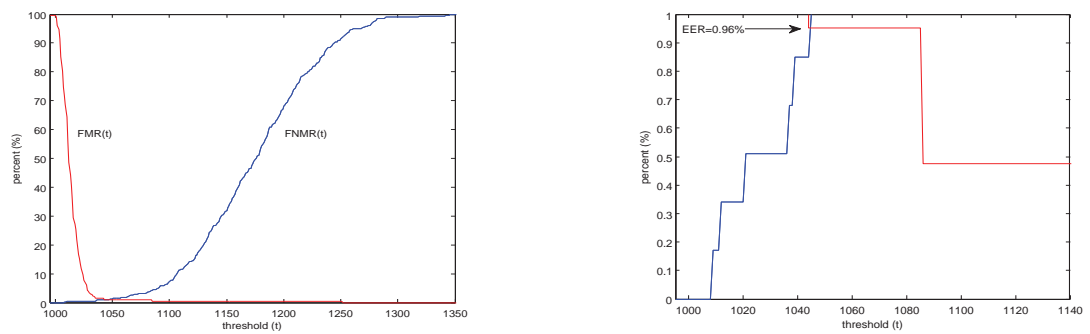

Fig. 15. left: Proposed method FNMR and FMR vs matching threshold $t$ on University of Bologna database right: Close up illustrating the EER (0.96\%).

FVC2002 competition, considering that the feature set was not in pristine condition due to the chosen extraction and filtering methods, highlighting the overall robustness of the proposed algorithm.

In addition, we improved the performance of the algorithm substantially over the enhanced shape context on both public datasets, despite using parameters only tuned for the FVC2002 database. Finally, all known competing matching algorithms tested on the University of Bologna database were beaten by the proposed fingerprint matching method. 

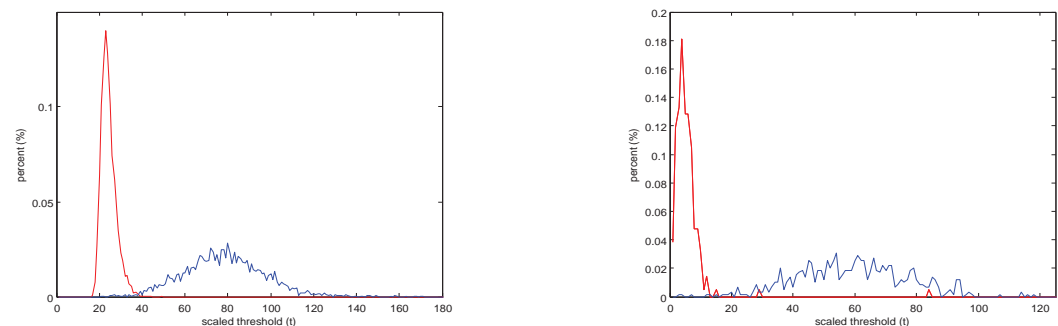

Fig. 16. Genuine (blue) and imposter (red) distributions for the left: FVC2002 Db1 set A database, and right: University of Bologna database.

\begin{tabular}{|l|c|}
\hline Matching Algorithm & EER (\%) \\
\hline CBFS Chikkerur \& Govindaraju (2006) & 1.50 \\
\hline TPS based Kwon et al. (2006) & 0.92 \\
\hline Meshgrid based Kwon et al. (2007) & 0.82 \\
\hline Hybrid Spiral based Shi \& Govindaraju (2009) & 1.98 \\
\hline PA08 Maio et al. (2002) $\left(8^{\text {th }}\right.$ place) & 0.98 \\
\hline PB35 Maio et al. (2002) $\left(5^{\text {th }}\right.$ place) & 0.61 \\
\hline PA15 Maio et al. $(2002)\left(1^{\text {st }}\right.$ place) & 0.1 \\
\hline Proposed method & $\mathbf{0 . 7 5}$ \\
\hline
\end{tabular}

Table 2. Performance comparison of matching algorithms on FVC2002 Db1 Set A

\begin{tabular}{|l|c|}
\hline Matching Algorithm & EER (\%) \\
\hline WGHT/Orientation-based Tico (2001) Tico et al. (2002) & $1.07-1.97$ \\
\hline Mutual Information Liu et al. (2006) & 1.5 \\
\hline Delaunay Triangulation Wang \& Gavrilova (2006) & 5.1 \\
\hline Enhanced Shape Context Kwan et al. (2006) & 12.79 \\
\hline Proposed method & $\mathbf{0 . 9 6}$ \\
\hline
\end{tabular}

Table 3. Performance comparison of matching algorithms on University of Bologna database

The matlab source code for the proposed fingerprint matching algorithm can be found at the Matlab Central ( Abraham (2010)) website.

\section{References}

Abraham, J. (2010). Matlab code for fingerprint matching algorithm.

URL: http://www.mathworks.com/matlabcentral/fileexchange/29280-fingerprint-matching -algorithm-using-shape-context-and-orientation-descriptors

Bazen, A. M. \& Gerez, S. H. (2003). Fingerprint matching by thin-plate spline modelling of elastic deformations, Pattern Recognition 36(8): 1859-1867.

Belongie, S., Malik, J. \& Puzicha, J. (2000). Shape context: A new descriptor for shape matching and object recognition, Neural Information Processing Systems Conference (NIPS), pp. 831-837. 
Belongie, S., Malik, J. \& Puzicha, J. (2002). Shape matching and object recognition using shape contexts, IEEE Transactions on Pattern Analysis and Machine Intelligence 24(4): 509-522.

Benhammadi, F., Amirouche, M., Hentous, H., Bey Beghdad, K. \& Aissani, M. (2007). Fingerprint matching from minutiae texture maps, PR 40(1): 189-197.

Bologna (2000). Biometric system laboratory: www2.csr.unibo.it/research/biolab, 168 fingerprint database (21 fingers 8 impressions).

Bookstein, F. L. (1989). Principal warps: Thin-plate splines and the decomposition of deformations, IEEE Trans. Pattern Anal. Mach. Intell. 11(6): 567-585.

Chikkerur, S. \& Govindaraju, V. (2006). K-plet and CBFS: A graph based fingerprint representation and matching algorithm, International Conference on Biometrics (accepted).

Chikkerur, S. \& Ratha, N. (2005). Impact of singular point detection on fingerprint matching performance, Automatic Identification Advanced Technologies, IEEE Workshop on 1: 207-212.

Chikkerur, S., Wu, C. \& Govindaraju, V. (2004). A systematic approach for feature extraction in fingerprint images, Biometric Authentication LNCS 3072: 344-350.

Farina, A., Kovacs Vajna, Z. \& Leone, A. (1999). Fingerprint minutiae extraction from skeletonized binary images, PR 32(5): 877-889.

Gonzalez, R. C. \& Woods, R. E. (2007). Digital Image Processing (3rd Edition), Prentice Hall.

Hatano, T., Adachi, T., Shigematsu, S., Morimura, H., Onishi, S., Okazaki, Y. \& Kyuragi, H. (2002). A fingerprint verification algorithm using the differential matching rate, Pattern Recognition, International Conference on 3: 30799.

Hong, L., Wan, Y. \& Jain, A. (1998). Fingerprint image enhancement: Algorithm and performance evaluation, "IEEE Transactions on Pattern Analysis and Machine Intelligence" 20(8): 777-789.

Jiang, X. \& Yau, W.-Y. (2000). Fingerprint minutiae matching based on the local and global structures, Pattern Recognition, International Conference on 2: 6038.

Jirachaweng, S., Leelasawassuk, T. \& Areekul, V. (2009). Performance and computational complexity comparison of block-based fingerprint enhancement, ICB09, pp. 656-665.

Jonker, R. \& Volgenant, A. (1987). A shortest augmenting path algorithm for dense and sparse linear assignment problems, Computing 38(4): 325-340.

Julasayvake, A. \& Choomchuay, S. (2007). An algorithm for fingerprint core point detection, Proc. of International Symposium on Signal Processing and its Applications 2007 (ISSPA-2007) 1(1): 1-4.

Kisel, A., Kochetkov, A. \& Kranauskas, J. (2008). Fingerprint minutiae matching without global alignment using local structures, Informatica 19(1): 31-44.

Kwan, P. W., Gao, J. \& Guo, Y. (2006). Fingerprint matching using enhanced shape context, Proceedings of The 21st Image and Vision Computing New Zealand (IVCNZ 2006) Great Barrier Island, New Zealand 1(1): 115-120.

Kwon, D., Yun, I. D., Kim, D. H. \& Lee, S. U. (2006). Fingerprint matching method using minutiae clustering and warping, Pattern Recognition, International Conference on 4: 525-528.

Kwon, D., Yun, I. D. \& Lee, S. U. (2007). A robust warping method for fingerprint matching, Computer Vision and Pattern Recognition, IEEE Computer Society Conference on 0: 1-6.

Liang, X. \& Asano, T. (2006). Fingerprint matching using minutia polygons, ICPR06, pp. I: 1046-1049.

Lindoso, A., Entrena, L., Liu Jimenez, J. \& San Millan, E. (2007). Correlation-based fingerprint matching with orientation field alignment, ICB07, pp. 713-721. 
Liu, L., Jiang, T., Yang, J. \& Zhu, C. (2006). Fingerprint registration by maximization of mutual information, IP 15(5): 1100-1110.

Maio, D. \& Maltoni, D. (1997). Direct gray-scale minutiae detection in fingerprints, PAMI 19(1): 27-40.

Maio, D., Maltoni, D., Cappelli, R., Wayman, J. \& Jain, A. K. (2002). "fvc2002: Second fingerprint verification competition", pp. 811-814.

Nanni, L. \& Lumini, A. (2009). Descriptors for image-based fingerprint matchers, Expert Systems with Applications 36(10): 12414-12422. Cited By (since 1996): 4.

URL: www.scopus.com

Qi, J., Wang, Y., Shi, Z., Xu, K. \& Zhao, X. (2004). Fingerprint matching integrating the global orientation field with minutia, ICBA, pp. 337-343.

Ratha, N. K. \& Bolle, R. (2003). Automatic Fingerprint Recognition Systems, SpringerVerlag.

Reisman, J., Jain, A. \& Ross, A. (2002). A hybrid fingerprint matcher, ICPR02, pp. III: 795-798.

Rutovitz, D. (1966). "pattern recognition", "J. Royal Statist. Soc" (129): 504-530.

Shi, Z. \& Govindaraju, V. (2009). Robust fingerprint matching using spiral partitioning scheme, ICB '09: Proceedings of the Third International Conference on Advances in Biometrics, Springer-Verlag, Berlin, Heidelberg, pp. 647-655.

Stoney, D. (1988). Distribution of epidermal ridge minutiae, Am. J. Physical Anthropology 77: 367-376.

Tian, L., Chen, L. \& Kamata, S.-i. (2007). Fingerprint matching using dual hilbert scans, SITIS '07: Proceedings of the 2007 Third International IEEE Conference on Signal-Image Technologies and Internet-Based System, IEEE Computer Society, Washington, DC, USA, pp. 593-600.

Tico, M. (2001). On Design and Implementation of Fingerprint-Based Biometric Systems, PhD thesis, PhD thesis, Tampere Univ. of Technology, Tampere, Finland.

Tico, M. \& Kuosmanen, P. (2003). Fingerprint matching using an orientation-based minutia descriptor, IEEE Transactions on Pattern Analysis and Machine Intelligence 25(8): 1009-1014.

Tico, M., Onnia, V. \& Kuosmanen, P. (2002). Fingerprint image enhancement based on second directional derivative of the digital image, EURASIP J. Appl. Signal Process. 2002(1): 1135-1144.

Wahba, G. (1990). Spline Models for Observational Data, SIAM.

Wang, C. \& Gavrilova, M. L. (2006). Delaunay triangulation algorithm for fingerprint matching, ISVD '06: Proceedings of the 3rd International Symposium on Voronoi Diagrams in Science and Engineering, IEEE Computer Society, Washington, DC, USA, pp. 208-216.

Xiao, Q. \& Raafat, H. (1991). Fingerprint image post-processing: A combined statistical and structural approach, PR 24(10): 985-992.

Yager, N. \& Amin, A. (2005). Coarse fingerprint registration using orientation fields, JASP 2005(13): 2043-2053.

Yang, J. C. \& Park, D.-S. (2008). A fingerprint verification algorithm using tessellated invariant moment features, Neurocomputing 71(10-12): 1939-1946.

Youssif, A. A., Chowdhury, M. U., Ray, S. \& Nafaa, H. Y. (2007). Fingerprint recognition system using hybrid matching techniques, "Computer and Information Science, ACIS International Conference on" 0: 234-240.

Zhang, W. \& Wang, Y. (2002). Core-based structure matching algorithm of fingerprint verification, Pattern Recognition, International Conference on 1: 10070.

Zhao, F. \& Tang, X. (2007). Preprocessing and postprocessing for skeleton-based fingerprint minutiae extraction, PR 40(4): 1270-1281. 


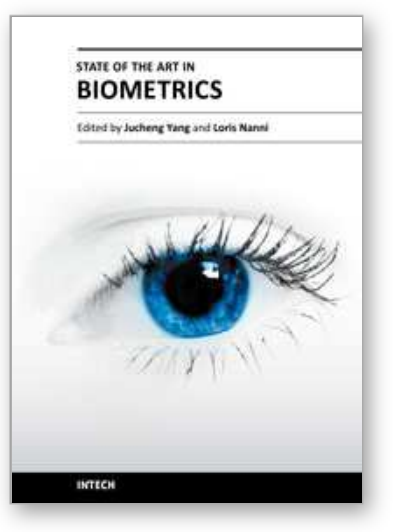

\author{
State of the art in Biometrics \\ Edited by Dr. Jucheng Yang
}

ISBN 978-953-307-489-4

Hard cover, 314 pages

Publisher InTech

Published online 27, July, 2011

Published in print edition July, 2011

Biometric recognition is one of the most widely studied problems in computer science. The use of biometrics techniques, such as face, fingerprints, iris and ears is a solution for obtaining a secure personal identification. However, the â€œoldâ€ biometrics identification techniques are out of date. This goal of this book is to provide the reader with the most up to date research performed in biometric recognition and descript some novel methods of biometrics, emphasis on the state of the art skills. The book consists of 15 chapters, each focusing on a most up to date issue. The chapters are divided into five sections- fingerprint recognition, face recognition, iris recognition, other biometrics and biometrics security. The book was reviewed by editors Dr. Jucheng Yang and Dr. Loris Nanni. We deeply appreciate the efforts of our guest editors: Dr. Girija Chetty, Dr. Norman Poh, Dr. Jianjiang Feng, Dr. Dongsun Park and Dr. Sook Yoon, as well as a number of anonymous reviewers

\title{
How to reference
}

In order to correctly reference this scholarly work, feel free to copy and paste the following:

Joshua Abraham, Paul Kwan and Junbin Gao (2011). Fingerprint Matching using A Hybrid Shape and Orientation Descriptor, State of the art in Biometrics, Dr. Jucheng Yang (Ed.), ISBN: 978-953-307-489-4, InTech, Available from: http://www.intechopen.com/books/state-of-the-art-in-biometrics/fingerprint-matchingusing-a-hybrid-shape-and-orientation-descriptor

\section{INTECH}

open science | open minds

\author{
InTech Europe \\ University Campus STeP Ri \\ Slavka Krautzeka 83/A \\ 51000 Rijeka, Croatia \\ Phone: +385 (51) 770447 \\ Fax: +385 (51) 686166 \\ www.intechopen.com
}

\author{
InTech China \\ Unit 405, Office Block, Hotel Equatorial Shanghai \\ No.65, Yan An Road (West), Shanghai, 200040, China \\ 中国上海市延安西路65号上海国际贵都大饭店办公楼 405 单元 \\ Phone: +86-21-62489820 \\ Fax: $+86-21-62489821$
}


(C) 2011 The Author(s). Licensee IntechOpen. This chapter is distributed under the terms of the Creative Commons Attribution-NonCommercialShareAlike-3.0 License, which permits use, distribution and reproduction for non-commercial purposes, provided the original is properly cited and derivative works building on this content are distributed under the same license. 